\title{
Polarizable interaction potential for water from coupled cluster calculations. II. Applications to dimer spectra, virial coefficients, and simulations of liquid water
}

\author{
Robert Bukowski, ${ }^{1}$ Krzysztof Szalewicz, ${ }^{1, a)}$ Gerrit C. Groenenboom, ${ }^{2}$ and \\ Ad van der Avoird ${ }^{2, b)}$ \\ ${ }^{1}$ Department of Physics and Astronomy, University of Delaware, Newark, Delaware 19716, USA \\ ${ }^{2}$ Theoretical Chemistry, Institute for Molecules and Materials, Radboud University Nijmegen, \\ Toernooiveld 1, 6525 ED Nijmegen, The Netherlands
}

(Received 13 September 2007; accepted 14 December 2007; published online 7 March 2008)

\begin{abstract}
The six-dimensional CC-pol interaction potential for the water dimer was used to predict properties of the dimer and of liquid water, in the latter case after being supplemented by a nonadditive potential. All the results were obtained purely from first principles, i.e., without any fitting to experimental data. Calculations of the vibration-rotation-tunneling levels of $\left(\mathrm{H}_{2} \mathrm{O}\right)_{2}$ and $\left(\mathrm{D}_{2} \mathrm{O}\right)_{2}$, a very sensitive test of the potential surface, gave results in good agreement with experimental high-resolution spectra. Also the virial coefficients and properties of liquid water agree well with measured values. The present model performs better than published force fields for water in a simultaneous reproduction of experimental data for dimer spectra, virials, and properties of the liquid. (C) 2008 American Institute of Physics. [DOI: 10.1063/1.2832858]
\end{abstract}

\section{INTRODUCTION}

To understand the properties of water clusters and of bulk water, one needs to know water's force field, i.e., the interaction potential. This potential can be represented as a many-body expansion beginning with the pair potential. The remaining, pairwise nonadditive contributions form a hierarchy of three-body, four-body, and higher-body interactions. ${ }^{1}$ The pair interactions provide by far the largest contribution to the total interaction energy for all types of clusters, ${ }^{1-6}$ but the nonadditive contributions have to be included in any accurate calculation for most systems. For liquid water, the pair interactions contribute about $85 \%$ to the total interaction energy at ambient conditions. ${ }^{2}$ Any work aimed at the determination of the "true" water force field has to build such a force field from many-body components which are individually correct. This approach should be contrasted with that adopted by the developers of empirical pair potentials for water $^{7-10}$ fitted in Monte Carlo (MC) or molecular dynamics (MD) simulations of liquid water to reproduce measured bulk properties. Such pair potentials include in some effective way the nonadditive effects, but the price paid for this is a very poor description of dimer spectra ${ }^{11-13}$ and virial coefficients. ${ }^{14}$ Therefore, despite their ability of predicting the bulk properties of water very well, one cannot consider these potentials to represent the true water force field. Moreover, improvements of the empirical potentials do not necessarily bring them closer to this goal. By contrast, the potentials resulting from $a b$ initio calculations only (without using any experimental data in their development) should converge to the true water force field as the quality of such calculations improves. This convergence should be tested on quan-

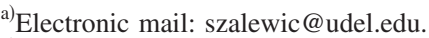

${ }^{b)}$ Electronic mail: avda@theochem.ru.nl.
}

tities as diverse as the dimer spectrum and the liquid properties. This is a safer and intrinsically better way of determining the true water force field than fitting the potentials to experimental data. One can avoid the many-body expansion of the interaction potential by performing "on the fly" calculations for all the molecules involved in a simulation, ${ }^{15-17}$ but this approach has its disadvantages and cannot at present provide reliable predictions for the complete range of systems from the water dimer to liquid water, see the discussion in Ref. 18 and later in the present paper.

The first component of the many-body expansion, the pair potential, can be tested directly on properties that depend exclusively on this potential, such as dimer spectra and virial coefficients. A large number of water dimer microwave and far-infrared transitions have been measured in supersonic molecular beams already in the $1990 \mathrm{~s}^{19-25}$ The comparison of the computed and measured spectra provides the most stringent test of the accuracy of a pair potential. On the other hand, accurate theoretical spectra can help to interpret the measured ones. ${ }^{26-28}$ The second virial coefficient is important for an accurate thermodynamic description of steam and in the construction of humidity standards. Accurate measurements are available for this quantity. ${ }^{29}$ Comparisons of theoretical and experimental virial coefficients allow one to evaluate the accuracy of the overall well depth of the potential and therefore provide information complementary to that coming from comparisons of spectra. Conversely, the theoretical input is important in the analysis of raw measurements that lead to virial coefficients and the determination of Ref. 29 was guided by the $a b$ initio SAPT-5s potential. ${ }^{30}$

The true pair potentials for water (and for much larger monomers, see, e.g., Refs. 31-33) can be obtained by $a b$ initio calculations. ${ }^{14,30,34-40}$ For water and some other small molecules, true pair potentials can also be obtained empiri- 
cally, by fitting to the dimer spectra. The first such water potential, denoted as VRT(ASP-W), was developed by Fellers et $a l^{41}$ This potential was obtained by explicitly refitting a number of parameters of the ASP-W surface ${ }^{34}$ to the vibration-rotation-tunneling (VRT) spectroscopic transitions. Recently, two new fits of this kind have been obtained by Goldman et $a l{ }^{42}$ dubbed VRT(ASP-W)II and VRT(ASPW)III. The same group of authors have developed ${ }^{43-45}$ also an empirical potential using a modified Matsuoka, Clementi, and Yoshimine ${ }^{46}$ (MCY) potential form. This potential was named VRT(MCY-5f), where " $\mathrm{f}$ " indicates that the potential assumes flexible monomers. In contrast to the published water pair potentials - both $a b$ initio and empirical - that were investigated in Refs. 11, 12, and 47, and all predicted the dimer spectra poorly, the VRT(ASP-W) potential was able to fit the spectra very well. However, a new purely $a b$ initio potential developed at about the same time, SAPT-5s, ${ }^{26,30}$ gave spectra almost as close to the measured ones as did VRT(ASP-W), in near quantitative agreement with experiment. $^{26-28}$ The remaining deviations from experiment were further reduced by "tuning" SAPT-5s, i.e., refitting the $a b$ initio data while subjecting the fit parameters to just a single constraint enforcing the desired splitting between two of the dimer energy levels. The "tuned" potential was dubbed SAPT-5st. ${ }^{28}$ SAPT-5st predicted the water dimer spectra even better than the empirical VRT potentials. ${ }^{44}$ Also the virial coefficients were recovered very well. Thus, it appears that within the past few years significant progress has been made towards creation of a very accurate water pair potential, as indicated by the predictions of the dimer properties. However, not all the newer potentials pass the dimer test. For example, the potential of Ref. 39 produces poor spectra. ${ }^{13}$

With the arrival of accurate water pair potentials, it became compelling to apply such potentials in simulations of liquid water. The leading nonadditive contribution to the force field required for such simulations was developed by Mas et l. $^{48}$ using the three-body version of SAPT (Ref. 49) at the Hartree-Fock (HF) level of theory. A physically motivated 12-dimensional site-site formula was fitted to $a b$ initio nonadditive energies of more than 7500 water trimers. To our knowledge, the resulting three-body potential $3 \mathrm{~B}(\mathrm{SAPT})$ is the unique such surface to date since it accounts not only for the induction (polarization) nonadditive effects, but also for the short-range ones that originate from electron exchanges. The adequacy of the HF level of theory for the three-body nonadditive component has been tested by calculating the three-dimensional torsional spectrum of the water trimer, ${ }^{26}$ where the SAPT-5s potential was used as the two-body component, and the HF three-body energies were calculated on a grid of trimer geometries defined by the dynamical calculation. The spectra from this calculation came out very close to the measured ones and surpassed in accuracy the calculations performed with other potentials, including the VRT(ASP-W) potential, indicating that the combined SAPT-5s $+3 \mathrm{~B}(\mathrm{SAPT})$ surface is a rather faithful representation of interactions within the water trimer. Note that the treatment of nonadditive interactions at the HF level of theory would be completely inadequate for other systems such as trimers involving rare-gas atoms. ${ }^{3-6}$
With the two- and three-body potentials of spectroscopic accuracy at hand, one can probe the properties of clusters and of bulk water. One can also address the fundamental question of the convergence of the many-body expansion of the interaction energy. Such investigations have until recently been restricted to rare gases. ${ }^{1,4,50}$ Liquid-phase simulations with the SAPT-5s+3B(SAPT) potential were performed in Ref. 2. The higher-than-three-body induction effects were also partly accounted for by means of an asymptotic polarization model. The simulations showed that the three-body nonadditive interactions play a crucial role in determining the structure of the liquid-in particular the number of hydrogen bonds. The results of the simulations were in overall good agreement with experiment. In particular, the oxygen-hydrogen and hydrogen-hydrogen pair distribution functions were closer to experiment than the results of most literature simulations. However, the oxygen-oxygen radial distribution function did not possess some features characteristic for the tetrahedral arrangement of water molecules, known from the neutron and x-ray diffraction experiments. ${ }^{51,52}$ This was the case even after the $N>$ three-body effects were included. In fact, the latter effects appeared to have a small impact on the properties of the liquid.

More recently, the VRT(ASP-W) potentials have also been used in simulations of liquid water. ${ }^{53}$ However, the nonadditive forces were represented only by the asymptotic polarization model. The resulting radial distribution functions were better than those produced by SAPT- $5 \mathrm{~s} / 5 \mathrm{st}$ in terms of the features related to the tetrahedral arrangements, but some other features differed from experiment rather substantially. The VRT(MCY-5f) potential has not yet been used in molecular simulations, but its rigid-monomer version, VRT(MCY-5r), gave significantly poorer agreement with experiment than achieved by VRT(ASP)III.

Clearly, the accurate water dimer potentials developed within the last few years ${ }^{30,42,43}$ led to somewhat disappointing results for liquid water. For several reasons, even an $e x-$ act water pair potential may poorly predict the properties of the liquid phase. ${ }^{1,2}$ The first suspected source of errors is the convergence of the many-body expansion. Although the convergence observed in Ref. 2 was fairly fast, this could be due to an insufficiently adequate representation of the nonadditive effects. On the other hand, studies on clusters (see the discussion in Refs. 1 and 2) also indicate a rapid convergence. Other possible sources of the discrepancies with experiment could be the monomer flexibility effects and quantum effects neglected in the molecular simulations. ${ }^{1,2}$ The possible inaccuracies of the two-body potential were initially considered to be a less likely reason for the discrepancies due to the excellent performance of this potential for dimer and trimer spectra and the virial coefficients. However, the improved predictions for liquid water obtained with the recent two-body potential ${ }^{35}$ computed using the SAPT(DFT) method, i.e., SAPT based on the density-functional theory (DFT) description of monomers ${ }^{54-56}$ - a potential developed to test the performance of the SAPT(DFT) approach rather than the properties of water-indicated that the two-body components may actually be the main culprit. 
To check this possibility, we have developed a pair potential for water ${ }^{13,18}$ using higher levels of theory and larger basis sets than in previous calculations. ${ }^{14,30}$ To reach as high accuracy as possible, the coupled-cluster method with single, double, and noniterative triple excitations $[\operatorname{CCSD}(\mathrm{T})]$ was applied. The calculations were also performed using manybody perturbation theory (MBPT) at the second-order level (MBPT2) based on the Møller-Plesset partition of the Hamiltonian (therefore often denoted as the MP2 method). The MBPT2 interaction energies were extrapolated to the complete basis set (CBS) limit from calculations in augmented triple-zeta and quadruple-zeta quality basis sets supplemented by bond functions. The contributions from the $\operatorname{CCSD}(\mathrm{T})$ calculations in the triple-zeta quality basis set which are beyond the MBPT2 level of theory were then added to the MBPT2/CBS energies. All the supermolecular interaction energies had the basis set superposition error removed using the counterpoise correction. ${ }^{57-61}$ The monomers were assumed rigid at a geometry averaged over the ground rovibrational state, which was shown to be the optimal choice for rigid-monomer approximations. ${ }^{62,63}$ The $a b$ initio calculations were performed for the same set of 2510 grid points on the dimer interaction energy surface as used to develop the SAPT-5s potential in Ref. 30. These points were fitted by an analytic, six-dimensional function similar to that used for SAPT-5s, except that it contains an induction term in the form of the classical polarization model. This makes it possible to straightforwardly include asymptotic many-body induction effects in calculations for clusters or condensed phases. The potential was referred to as CC-pol. ${ }^{13}$

The CC-pol potential was applied in Ref. 18 to predict the characteristic points on the water dimer potential energy surface. These points were found to be very close to those obtained in accurate direct optimizations of all coordinates by Tschumper et al. ${ }^{64}$ This is not surprising since the level of theory used in the development of the CC-pol potential was very similar to that applied in Ref. 64. Thus, the discrepancies are mainly due to the rigid-monomer approximation employed in CC-pol and to inaccuracies of CC-pol relative to the computed $a b$ initio interaction energies. One should emphasize here that our calculations have reached this high accuracy for the complete potential energy surface, whereas the calculations of Ref. 64 and similar earlier literature work examined only a few selected points on this surface.

The CC-pol potential has been applied in calculations of VRT spectra of the water dimer, calculations of virial coefficients, and in simulations of liquid water. The main results have been presented in Ref. 13. In the current paper, we supplement these results with important additional data. In particular, the calculations of VRT spectra have been extended to the deuterated water dimer, the isotope shifts of the second virial coefficients have been computed, and the deviations from experiment of both the positions and heights of the peaks have been presented for the pair distribution functions. Our results are analyzed from a different perspective than in Ref. 13 and more extensive comparisons with literature are made. We provide here a decomposition of the second virial coefficients into the classical and quantum components, the latter at two different levels. These results give the most precise information to date on the relevance of these quantum effects. The additional results of our MD simulations presented here allow to shed new light on the convergence of many-body expansion for liquid water. We also discuss the importance of monomer flexiblity and quantum effects on the properties of liquid water.

The outline of the paper is as follows. Section II discusses the computation of VRT spectra of the dimer, whereas Sec. III presents the results of the second virial coefficient calculations. In Sec. IV, we report the results of liquid water simulations with the CC-pol potential and reexamine the question of the convergence of many-body expansion of the interaction energy. Finally, Sec. V contains a summary and conclusions.

\section{VIBRATION-ROTATION-TUNNELING SPECTRUM OF WATER DIMER}

The water dimer has eight equivalent equilibrium structures related to one another by interchanging the labels of the two hydrogen atoms on either one of the two $\mathrm{H}_{2} \mathrm{O}$ (or $\mathrm{D}_{2} \mathrm{O}$ ) monomers, and by an interchange of the two monomers. In other words, all interchanges of equivalent nuclei that do not break the chemical bonds within the two monomers are feasible, in the sense of Longuet-Higgins. ${ }^{65}$ These interchange operations, combined with inversion, generate the permutation-inversion (PI) symmetry group $G_{16}$ of the water dimer. The observed spectral splittings result from quantum mechanical tunneling between the eight equivalent minima on the global potential surface. Previous theoretical work ${ }^{28,41}$ has shown that the intermolecular vibrations of the water dimer cannot be considered as small amplitude nearly harmonic vibrations. Hence, the computation of the bound energy levels of the water dimer should involve a fully coupled treatment in all six intermolecular degrees of freedom, similar to a coupled-channel scattering calculation. Such a treatment has been presented in Refs. 28, 41, and 47. It was demonstrated in these papers that the resulting water dimer energy levels, through a comparison with the corresponding high accuracy experimental data, provide an extremely sensitive test of water pair potentials.

The formalism is explained in detail in Ref. 28. The angular basis of Wigner functions $D_{m k}^{(j)^{*}}$ that describe the internal rotations of monomers $\mathrm{A}$ and $\mathrm{B}$ was truncated at maximum values of $j_{A}$ and $j_{B}$ equal to 11 for the $\mathrm{H}_{2} \mathrm{O}$ dimer and 12 for the $\mathrm{D}_{2} \mathrm{O}$ dimer. The Wigner functions on the two monomers are Clebsch-Gordan coupled and multiplied by Wigner functions for the overall rotation of the complex with quantum numbers $J$, the total angular momentum of the complex, $M$, the projection of this angular momentum on a space-fixed axis, and $K$, the projection on the dimer $z$ axis. The latter axis coincides with the vector $\boldsymbol{R}$ that points from the center of mass of monomer A to that of monomer B. The quantum numbers $J$ and $M$ are exact quantum numbers. We neglected the off-diagonal Coriolis coupling between the internal angular momenta $\boldsymbol{j}_{A}, \boldsymbol{j}_{B}$ and the overall angular momentum $\boldsymbol{J}$, which also makes $K$ a good quantum number. The water dimer is a near (prolate) symmetric top and the offdiagonal Coriolis coupling leads only to a minute asymmetry 
doubling of the water dimer levels. For the radial coordinate $R$, the length of the vector $\boldsymbol{R}$, we used a discrete variable representation (DVR) with four contracted DVR functions obtained by using a one-dimensional radial cut through the equilibrium geometry in the six-dimensional potential surface, see Ref. 28. This basis, although small, gives well converged results. With the enlargement from three to four radial basis functions, the VRT levels hardly changed. In order to simplify the calculations and find the spectral selection rules, the basis is adapted to the irreducible representations (irreps) $A_{1}^{ \pm}, B_{1}^{ \pm}, A_{2}^{ \pm}, B_{2}^{ \pm}$, and $E^{ \pm}$of the PI group $G_{16}$.

Before discussing the results, we define some important parameters that characterize the spectra of the water dimer. The origins $o_{1}(0)$ and $o_{2}(0)$ are the average energy of the $A_{1}^{+}$ and $B_{1}^{+}$symmetry levels and the average energy of the $A_{2}^{-}$and $B_{2}^{-}$symmetry levels, respectively, for $J=K=0$. For $K \neq 0$, the levels occur in degenerate pairs, $A_{1}^{ \pm}, B_{1}^{\mp}$ and $A_{2}^{ \pm}, B_{2}^{\mp}$, due to our neglect of the off-diagonal Coriolis coupling. In the experimental spectra, these pairs are separated by minute energy differences. To be able to compare to data presented by experimental groups, we have followed their definition for the origins $o_{1}(K)$ and $o_{2}(K)$ of the $K>0$ levels. For a given symmetry, the origins $o(K)$ were obtained from the measured transition frequencies by fits of the initial and final $(J, K)$ levels to the formula ${ }^{24,45}$

$$
E(J, K)=o(K)+\frac{(B+C)}{2}\left[J(J+1)-K^{2}\right] .
$$

This equation was, in fact, supplemented with some small perturbation terms, but for our low $J$ values, we may neglect these terms. For $K=1$, the origins $o(1)$ are not simply the levels with $(J, K)=(1,1)$, but are shifted down by $(B+C) / 2$ relative to these levels. The $K=1$ rotational constant $B+C$ was obtained by us from the difference between $K=1$ levels with $J=2$ and $J=1$. The constants are only slightly different (of the order of $0.001 \mathrm{~cm}^{-1}$ ) and we have averaged them over all states split by tunneling. The averaged origins $o_{1}(K)$ and $o_{2}(K)$ are defined for each value of $K$ and were computed as the arithmetic averages of the origins defined by Eq. (1) for the states $A_{1}^{+}, B_{1}^{+}$and $A_{2}^{-}, B_{2}^{-}$, respectively. [Note that in Refs. 28 and 35 the theoretical $K=1$ origins were defined differently - as extrapolations of the levels with $J \geqslant K=1$ to $J=0$ - and therefore were not corresponding to the experimental definition. These origins of Refs. 28 and 35 are lower than the present ones by $(B+C) / 2]$.

The largest tunneling splitting, denoted by $a(K)$, is the so-called acceptor splitting, which is strongly $K$ dependent. It is caused by an interchange of the two hydrogen atoms of the hydrogen bond acceptor monomer. The two corresponding minima in the potential surface are separated by the lowest energy barrier in the potential surface, saddle point No. 2 in Tables II and III of Ref. 18. The splitting $a(K)$ can be extracted from the (calculated or measured) spectra as the energy difference between the origins $o_{2}(K)$ and $o_{1}(K)$. The interchange splittings, denoted by $i_{1}$ and $i_{2}$, correspond to the interchange of the donor and acceptor molecules. The barrier that separates the two corresponding minima in the potential surface is saddle point No. 4 in Tables II and III of Ref. 18. These tunneling splittings can be extracted from the spectra as well: $i_{1}$ is the difference between the $B_{1}^{ \pm}$and $A_{1}^{ \pm}$levels and $i_{2}$ is the difference between the $B_{2}^{\overline{+}}$ and $A_{2}^{\overline{+}}$ levels. The levels of $E^{ \pm}$symmetry are always between the $A_{1}$ and $B_{1}$ levels and between the $A_{2}$ and $B_{2}$ levels of the same \pm parity. The small shifts of the energies of the $E^{ \pm}$levels with respect to the average energies of the $A_{1}^{ \pm}$and $B_{1}^{ \pm}$levels and the $A_{2}^{ \pm}$ and $B_{2}^{ \pm}$levels are not considered here. Calculations on different water pair potentials ${ }^{47}$ have shown that the tunneling splittings depend very sensitively on the heights and shapes of the barriers in the potential surface.

Other quantities discussed are the end-over-end rotational constant $B+C$ and the much larger rotational constant $A$ for rotation about the prolate axis (nearly coinciding with the vector $\boldsymbol{R}$ ). Similarly as for $K=1$, the value of $B+C$ for $K=0$ was obtained as the difference between levels with $J=1$ and $J=0$. Then, from the usual prolate near-symmetric top expression

$$
E(J, K)=E(0,0)+\frac{(B+C)}{2}\left[J(J+1)-K^{2}\right]+A K^{2}
$$

and the definition of the origins in Eq. (1), it follows that $A$ is simply the difference between the $K=1$ and $K=0$ origins. Here we applied this formula to the averaged origins $\left[o_{1}(K)+o_{2}(K)\right] / 2$. Note that for $\left(\mathrm{H}_{2} \mathrm{O}\right)_{2}$ we took the experimental value $A=7.44 \mathrm{~cm}^{-1}$ extracted from the microwave spectrum, ${ }^{20}$ whereas in Ref. 28 the value $A=7.59 \mathrm{~cm}^{-1}$ from terahertz spectra ${ }^{25}$ was given.

The properties of the states with $K=0$ and $K=1$ calculated on the CC-pol potential are listed and compared with the available experimental data in Tables I and II for the $\mathrm{H}_{2} \mathrm{O}$ dimer and the $\mathrm{D}_{2} \mathrm{O}$ dimer, respectively. A subset of the $\mathrm{H}_{2} \mathrm{O}$ data has been graphically presented in Ref. 13. The dissociation energies are $D_{0}=1111 \mathrm{~cm}^{-1}$ for the $\mathrm{H}_{2} \mathrm{O}$ dimer and $D_{0}$ $=1255 \mathrm{~cm}^{-1}$ for the $\mathrm{D}_{2} \mathrm{O}$ dimer, while the minimum in the fit of the CC-pol potential corresponds to $D_{e}=5.097 \mathrm{kcal} / \mathrm{mol}$ $=1783 \mathrm{~cm}^{-1}$. Experimentally, $D_{0}$ has not been accurately determined. The calculated and measured ground state tunneling levels of the $\mathrm{H}_{2} \mathrm{O}$ dimer and the $\mathrm{D}_{2} \mathrm{O}$ dimer are displayed in Figs. 1 and 2, respectively. The rotationally excited levels with $J>K$ are not drawn since these almost coincide with the $J=K$ levels [these levels are shifted only by $(B+C) J$ for $J=K+1]$. The quantity $\Delta$ shown in Figs. 1 and 2 and used to compute the rotational constant $A$ is the energy difference between the levels with $J=K=1$ and those with $J=K=0$, averaged over all symmetries $A_{1}^{+}, B_{1}^{+}, A_{2}^{-}, B_{2}^{-}$. The levels [origins $o_{1}(K)$ and $o_{2}(K)$ for $\left.K=0,1\right]$ corresponding to the intermolecular vibrations of the $\mathrm{H}_{2} \mathrm{O}$ dimer and the $\mathrm{D}_{2} \mathrm{O}$ dimer are shown in Figs. 3 and 4, respectively. The assignment of these intermolecular vibrations was discussed in Ref. 27, on the basis of the VRT wave functions calculated on the SAPT-5st potential surface. Note that the mode that was assigned to the in-plane bend fundamental in the earlier experimental and theoretical papers, on the basis of approximate harmonic oscillator calculations, is actually the donor torsion overtone.

Looking at the results for the $\mathrm{H}_{2} \mathrm{O}$ dimer in Table I and Fig. 1, we may conclude that the agreement between the ground state tunneling levels calculated on the CC-pol potential and the experimental data is remarkably good. It is 
TABLE I. VRT levels, tunneling splittings, and rotational constants (in $\left.\mathrm{cm}^{-1}\right)$ of $\left(\mathrm{H}_{2} \mathrm{O}\right)_{2}$ calculated from the CC-pol potential. The numbers in parentheses are experimental values from Refs. 20, 21, 25, 44, and 45. The assignment of the intermolecular vibrations is given in Ref. 27. When it deviates from the earlier assignment according to Ref. 25 , the latter is added in square brackets. The energy zero corresponds to $D_{0}$ $=1110.92 \mathrm{~cm}^{-1}$

\begin{tabular}{|c|c|c|c|c|c|c|c|}
\hline & $o_{1}$ & $\mathrm{O}_{2}{ }^{\mathrm{a}}$ & $a$ & $i_{1}$ & $i_{2}$ & $B+C$ & $A$ \\
\hline \multicolumn{8}{|c|}{ Ground state $\left(A^{\prime}\right)$} \\
\hline$K=0$ & $\begin{array}{c}0 \\
(0)\end{array}$ & $\begin{array}{c}13.28 \\
(11.18)\end{array}$ & $\begin{array}{c}13.28 \\
\mathrm{~b}\end{array}$ & $\begin{array}{c}0.69 \\
(0.75)\end{array}$ & $\begin{array}{c}0.66 \\
(0.65)\end{array}$ & $\begin{array}{c}0.4227 \\
(0.4112)\end{array}$ & \\
\hline$K=1$ & $\begin{array}{c}15.63 \\
(14.39)\end{array}$ & $\begin{array}{c}12.33 \\
(11.66)\end{array}$ & $\begin{array}{l}3.30 \\
\mathrm{~b}\end{array}$ & $\begin{array}{c}0.68 \\
(0.71)\end{array}$ & $\begin{array}{c}0.51 \\
(0.54)\end{array}$ & $\begin{array}{c}0.4226 \\
(0.4108)\end{array}$ & $\begin{array}{c}7.34 \\
(7.44)\end{array}$ \\
\hline \multicolumn{8}{|c|}{ Donor torsion $\left(A^{\prime \prime}\right)$} \\
\hline$K=0$ & 118.71 & $\begin{array}{c}60.48 \\
(64.52)\end{array}$ & 58.24 & 4.59 & $\begin{array}{c}1.50 \\
(2.54)\end{array}$ & 0.4246 & \\
\hline$K=1$ & $\begin{array}{c}84.79 \\
(87.75)\end{array}$ & 93.35 & 8.57 & $\begin{array}{c}0.42 \\
(1.11)\end{array}$ & 2.35 & $\begin{array}{c}0.4229 \\
(0.4083)\end{array}$ & -0.53 \\
\hline \multicolumn{8}{|c|}{ Acceptor wag $\left(A^{\prime}\right)$} \\
\hline$K=0$ & $\begin{array}{c}110.94 \\
(107.93)\end{array}$ & $\begin{array}{c}115.66 \\
(108.89)\end{array}$ & $\begin{array}{c}4.72 \\
(0.96)\end{array}$ & $\begin{array}{c}3.06 \\
(2.95)\end{array}$ & $\begin{array}{c}0.50 \\
(0.02)\end{array}$ & $\begin{array}{c}0.4220 \\
(0.4094)\end{array}$ & \\
\hline$K=1$ & $\begin{array}{c}113.99 \\
(109.98)\end{array}$ & $\begin{array}{c}128.65 \\
(123.56)\end{array}$ & $\begin{array}{c}14.66 \\
(13.58)\end{array}$ & $\begin{array}{c}6.56 \\
(5.24)\end{array}$ & $\begin{array}{c}5.08 \\
(3.41)\end{array}$ & $\begin{array}{c}0.4245 \\
(0.4122)\end{array}$ & $\begin{array}{c}8.02 \\
(8.08)\end{array}$ \\
\hline \multicolumn{8}{|c|}{ Acceptor twist $\left(A^{\prime \prime}\right)$} \\
\hline$K=0$ & 129.39 & $\begin{array}{c}117.76 \\
(120.19)\end{array}$ & 11.63 & 2.80 & $\begin{array}{c}9.89 \\
(9.39)\end{array}$ & $\begin{array}{c}0.4246 \\
(0.4138)\end{array}$ & \\
\hline$K=1$ & 144.63 & 135.79 & 8.85 & 4.80 & 6.21 & 0.4238 & 16.64 \\
\hline \multicolumn{8}{|c|}{ Donor torsion overtone $\left(A^{\prime}\right)$ [in-plane bend] } \\
\hline$K=0$ & 133.15 & $\begin{array}{c}156.92 \\
(153.62)\end{array}$ & 23.77 & 8.47 & $\begin{array}{l}1.75 \\
(1.877)\end{array}$ & 0.4179 & \\
\hline$K=1$ & 159.35 & 156.72 & 2.62 & 1.35 & 1.36 & 0.4129 & 13.00 \\
\hline \multicolumn{8}{|c|}{ Stretch $\left(A^{\prime}\right)$} \\
\hline$K=0$ & 150.13 & 189.58 & 39.45 & 2.42 & 18.34 & 0.4179 & \\
\hline
\end{tabular}

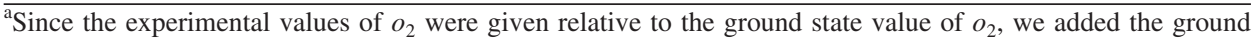
state acceptor splitting $a(K=0)=11.18 \mathrm{~cm}^{-1}$ predicted by the SAPT-5st potential of Ref. 28 to all experimental values.

${ }^{\mathrm{b}}$ Acceptor splitting $a(K=0)+a(K=1)=16.58 \mathrm{~cm}^{-1}$ (calculated) and $13.92 \mathrm{~cm}^{-1}$ (experimental).

better than with any previous $a b$ initio potential. Older purely $a b$ initio potentials, such as MCY, ASP-S, or ASP-W, gave much worse results for the water dimer tunneling splittings, ${ }^{13,41}$ as it can also be seen in Fig. 5 which shows the average percentage deviations from experiments for spectra computed from various potentials (for further comparisons of these spectra, see Refs. 13 and 35). Even recent $a b$ initio potentials, such as SAPT-5s and SDFT-5s, although substantially better than the older ones, produce energy levels that are clearly not as good as those from the CC-pol potential. Potentials that give a comparably good agreement with the experimental spectra as CC-pol have either been fitted to these spectra, such as the different versions of the VRT(ASP) potential ${ }^{11,42}$ and the VRT(MCY) potential, ${ }^{43}$ or were improved by a tuning procedure with the use of spectral data, such as SAPT-5st. ${ }^{28}$ The empirical potentials such as TIP4P (Ref. 8) produce spectra which are qualitatively different from experimental ones, as demonstrated by the very large errors seen in Fig. 5. The reason is the effective character of these potentials: the two-body interactions have to be unphysically distorted to approximately account for many- body effects. The recent TTM2.1 potential $^{39}$ has errors comparable to those of TIP4P.

The largest discrepancy of the CC-pol predictions with experiment is found for the acceptor splitting $a(K)$. Experimentally, the quantity $a(0)+a(1)$ was determined to be $13.92 \mathrm{~cm}^{-1}$, whereas calculations with the CC-pol potential gave $16.58 \mathrm{~cm}^{-1}$. All previous ab initio potentials, except for ASP-S, gave larger discrepancies for this quantity. The discrepancy that remains in the $\mathrm{CC}$-pol results is probably due to the fact that the monomer geometries are fixed in this potential. Calculations accounting for the effects of monomer flexibility ${ }^{43,68,69}$ have shown that the quantity $a(0)+a(1)$ decreases when the $\mathrm{H}_{2} \mathrm{O}$ monomers get the possibility to adapt their geometry to the intermolecular interactions. The interchange splittings $i_{1}$ and $i_{2}$ of the vibrational ground state and also the rotational constants $A$ and $B+C$ computed from CC-pol agree well with experiment. The fact that $B+C$ is too large by about $0.01 \mathrm{~cm}^{-1}$ indicates that the $R_{e}$ value of the CC-pol potential is slightly too small. The negative value of the constant $A$ for one of the states is a reflection of the significant deviation of the $\mathrm{H}_{2} \mathrm{O}$ dimer from a rigid rotor. 
TABLE II. VRT levels, tunneling splittings, and rotational constants $\left(\right.$ in $\left.\mathrm{cm}^{-1}\right)$ of $\left(\mathrm{D}_{2} \mathrm{O}\right)_{2}$ calculated from the CC-pol potential. The numbers in parentheses are experimental values from Refs. 19, 22-24, 44, and 45. The energy zero corresponds to $D_{0}=1255.29 \mathrm{~cm}^{-1}$.

\begin{tabular}{|c|c|c|c|c|c|c|c|}
\hline & $o_{1}$ & $o_{2}{ }^{a}$ & $a$ & $i_{1}$ & $i_{2}$ & $B+C$ & $A$ \\
\hline \multicolumn{8}{|c|}{ Ground state $\left(A^{\prime}\right)$} \\
\hline \multirow[t]{2}{*}{$K=0$} & 0 & 2.41 & 2.41 & 0.043 & 0.042 & 0.3804 & \\
\hline & (0) & $(1.77)$ & $(1.77)$ & $(0.039)$ & $(0.036)$ & $(0.3622)$ & \\
\hline \multirow[t]{2}{*}{$K=1$} & 5.77 & 4.98 & 0.79 & 0.042 & 0.038 & 0.3804 & 4.17 \\
\hline & $(5.36)$ & $(4.74)$ & $(0.62)$ & $(0.036)$ & $(0.033)$ & $(0.3621)$ & (4.17) \\
\hline \multicolumn{8}{|c|}{ Donor torsion $\left(A^{\prime \prime}\right)$} \\
\hline \multirow[t]{2}{*}{$K=0$} & 74.99 & 54.71 & 20.28 & 0.151 & 0.098 & 0.3789 & \\
\hline & (75.38) & $(59.59)$ & $(15.81)$ & $(0.328)$ & $(0.203)$ & $(0.3622)$ & \\
\hline \multirow[t]{2}{*}{$K=1$} & 63.78 & 69.07 & 5.29 & 0.046 & 0.136 & 0.3782 & 1.58 \\
\hline & $(68.27)$ & $(71.81)$ & $(3.54)$ & $(0.132)$ & $(0.257)$ & $(0.3600)$ & $(2.56)$ \\
\hline \multicolumn{8}{|c|}{ Acceptor wag $\left(A^{\prime}\right)$} \\
\hline \multirow[t]{2}{*}{$K=0$} & 82.81 & 88.39 & 5.58 & 0.174 & 0.219 & 0.3791 & \\
\hline & (82.64) & $(84.40)$ & $(1.77)$ & $(0.131)$ & $(0.112)$ & $(0.3603)$ & \\
\hline \multirow[t]{2}{*}{$K=1$} & 87.38 & 91.06 & 3.68 & 0.720 & 0.456 & 0.3813 & 3.62 \\
\hline & $(85.57)$ & $(89.56)$ & $(4.00)$ & $(0.398)$ & $(0.168)$ & $(0.3592)$ & $(4.05)$ \\
\hline \multicolumn{8}{|c|}{ Acceptor twist $\left(A^{\prime \prime}\right)$} \\
\hline \multirow[t]{2}{*}{$K=0$} & 89.68 & 87.62 & 2.06 & 0.852 & 1.041 & 0.3830 & \\
\hline & $(92.91)$ & $(90.37)$ & $(2.54)$ & $(0.432)$ & $(0.443)$ & $(0.3665)$ & \\
\hline$K=1$ & 98.58 & 94.85 & 3.74 & 0.530 & 0.757 & 0.3819 & 8.06 \\
\hline \multicolumn{8}{|c|}{ Donor torsion overtone $\left(A^{\prime}\right)$ [in-plane bend] } \\
\hline \multirow[t]{2}{*}{$K=0$} & 101.89 & 134.14 & 32.25 & 0.634 & 1.213 & 0.3793 & \\
\hline & $(104.24)$ & & & $(0.783)$ & & $(0.3632)$ & \\
\hline$K=1$ & 123.00 & 114.25 & 8.75 & 0.211 & 0.005 & 0.3791 & 0.61 \\
\hline \multirow[b]{2}{*}{$K=0$} & & Donor & on + accep & wag comb & on $\left(A^{\prime \prime}\right)$ & & \\
\hline & 137.14 & 138.04 & 0.90 & 0.077 & 0.587 & 0.3791 & \\
\hline \multicolumn{8}{|c|}{ Stretch $\left(A^{\prime}\right)$} \\
\hline$K=0$ & 137.73 & 147.16 & 9.42 & 3.163 & 2.441 & 0.3823 & \\
\hline
\end{tabular}

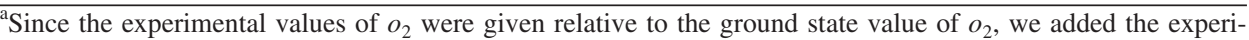
mental estimate (Ref. 23) for the ground state acceptor splitting $a(K=0)=53 \mathrm{GHz}=1.7679 \mathrm{~cm}^{-1}$ to all experimental values.

Table II and Fig. 2, which show the vibrational ground state levels for the $\mathrm{D}_{2} \mathrm{O}$ dimer, demonstrate that the CC-pol potential gives excellent tunneling splittings also for this system. Let us note here that the tunneling splittings become much smaller when $\mathrm{H}_{2} \mathrm{O}$ is replaced by $\mathrm{D}_{2} \mathrm{O}$, mainly because the rotational constants of heavy water are smaller by about a factor of 2 than those of normal water. The splittings are even more sensitive to the barriers in the potential surface than for the $\mathrm{H}_{2} \mathrm{O}$ dimer. The tunneling splittings from CCpol are considerably better than those from VRT(ASP-W)III, for which these splittings were used in the fit. The calculated acceptor splittings $a(K)$ for the $\mathrm{D}_{2} \mathrm{O}$ dimer are too large, just as for the $\mathrm{H}_{2} \mathrm{O}$ dimer, which probably has the same origin: the lack of monomer flexibility. Only potentials with parameters that were fitted to or improved with the help of experimental data gave better agreement with these data than CC-pol.

The comparison with experiment for the frequencies of the intermolecular vibrations in Tables I and II and in Figs. 3 and 4 further confirms the high quality of CC-pol. Figure 5 shows that for the $\mathrm{H}_{2} \mathrm{O}$ dimer CC-pol predicts frequencies of vibrational origins better than any other potential to date ex- cept for VRT(MCY-5f) ${ }^{43}$ which was fitted to these data. The CC-pol predictions are even better than those of other potentials fitted to spectra, such as VRT(ASP-W)III and SAPT-5st.

The largest and best defined set of experimental data is available for the $\mathrm{D}_{2} \mathrm{O}$ dimer. This set of data was used to fit the VRT(ASP-W)II and VRT(ASP-W)III potentials. ${ }^{42}$ For the $\mathrm{D}_{2} \mathrm{O}$ dimer, the origins of the intermolecular vibration bands disagree with the measurements by $4.1 \%$ on average for the CC-pol potential, whereas for the VRT(ASP-W)III potential, the better of the two empirical potentials, the average error is $4.3 \%$. For the SAPT-5st potential based on the $a b$ initio SAPT-5s potential, and tuned to the measured ground state tunneling splittings, the frequencies of the $\mathrm{D}_{2} \mathrm{O}$ intermolecular vibrations deviate from experiment by $5.8 \%$ on average. The VRT(MCY-5f) potential has not been used in calculations for the $\mathrm{D}_{2} \mathrm{O}$ dimer. The fact that the purely $a b$ initio CC-pol potential gives predictions for the $\mathrm{D}_{2} \mathrm{O}$ dimer even better than those from the empirical potentials is remarkable.

The overall performance of CC-pol can be judged best from Fig. 5. One can see that the predictions of CC-pol for the $\mathrm{H}_{2} \mathrm{O}$ dimer are better than those of all other potentials 


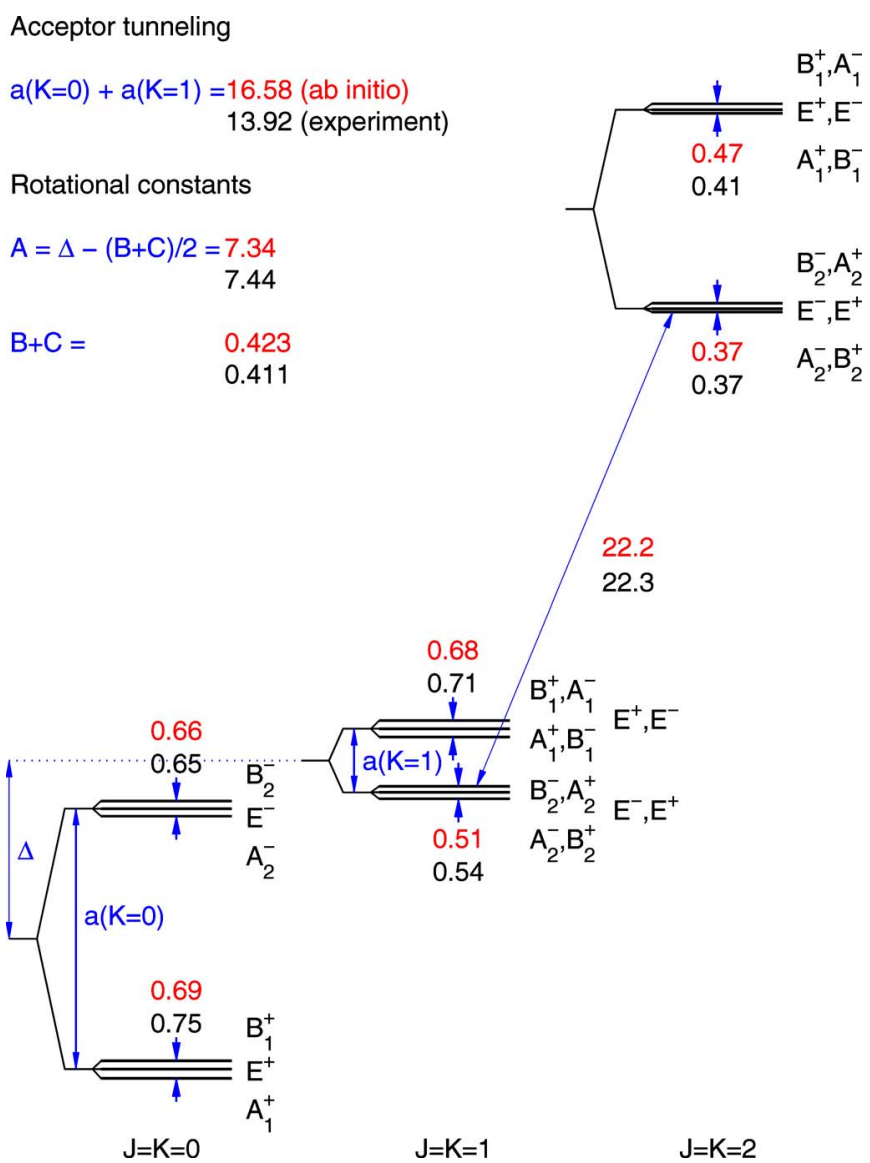

FIG. 1. (Color online) Ground state VRT levels of the $\mathrm{H}_{2} \mathrm{O}$ dimer (in $\mathrm{cm}^{-1}$ ) from converged calculations with the CC-pol ab initio potential, in comparison with experimental data (Refs. 20, 21, and 25) (lower numbers). The energies are drawn to scale (using experimental values).

except for VRT(MCY-5f) and SAPT-5st. The latter potential is overall closest to experiment, but even in this case the CC-pol predictions for the vibrational origins are slightly better, as already mentioned. Similarly, CC-pol gives better predictions for the rotational constants than does VRT(MCY-5f). Interestingly, all three features of CCpol spectrum are closer to experiment than those of VRT(ASP-W)III.

\section{SECOND VIRIAL COEFFICIENT}

The second virial coefficient of steam, $B(T)$, is a twobody property, depending only on the dimer interaction potential. The quality of such a potential can thus be judged by computing $B(T)$ and comparing the results to experiments. Theoretical calculations of $B(T)$ for water are complicated by the fact that due to the small moments of inertia of the water molecule, quantum contributions are quite large and cannot be neglected, especially at lower temperatures. The quantum contributions to $B(T)$ are most often included perturbatively, up to the first order in $\hbar^{2}$. The expression for the second virial coefficient at temperature $T$ then becomes (for rigid monomers) ${ }^{14,70,71}$
Acceptor tunneling

$a(K=0) \quad a(K=1) \quad a(K=2)$

$\begin{array}{llll}2.41 & 0.79 & 1.71 \quad \text { (ab initio) }\end{array}$

$\begin{array}{llll}1.77 & 0.62 & 1.31 & \text { (experiment) }\end{array}$

Rotational constants

$A=\Delta-(B+C) / 2=4.17$

4.17

$\mathrm{B}+\mathrm{C}=\quad 0.380$
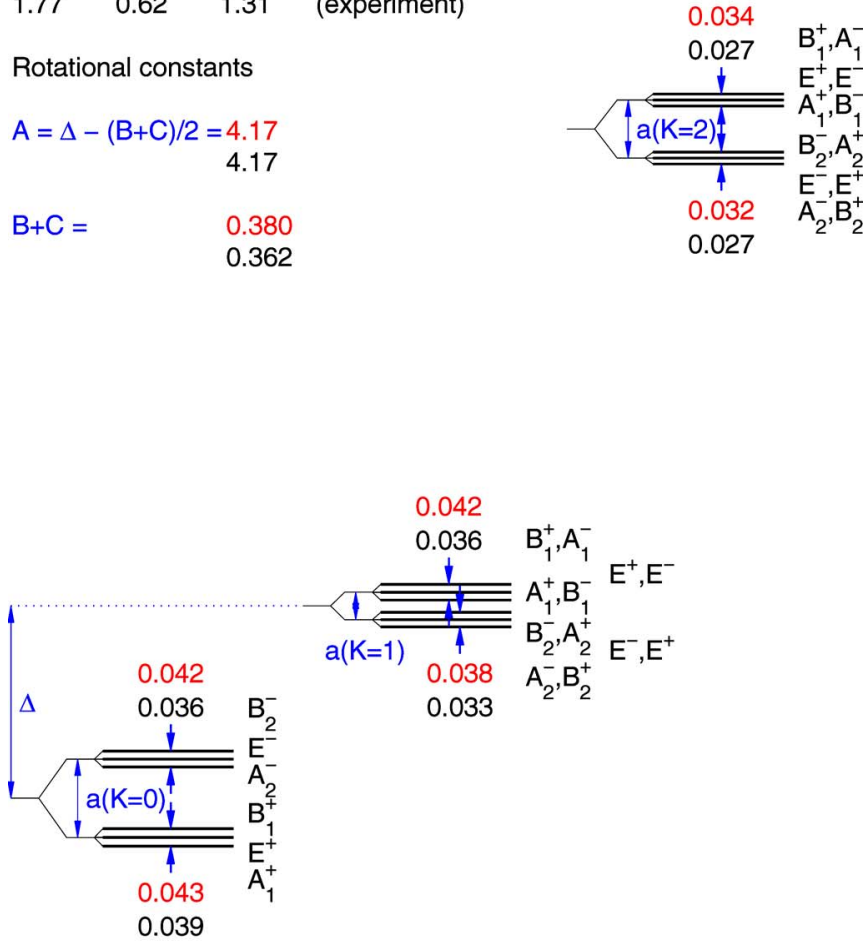

$\mathrm{J}=\mathrm{K}=0$

$\mathrm{J}=\mathrm{K}=1$

$\mathrm{J}=\mathrm{K}=2$

FIG. 2. (Color online) Ground state VRT levels of the $\mathrm{D}_{2} \mathrm{O}$ dimer $\left(\right.$ in $\mathrm{cm}^{-1}$ ) calculated from the CC-pol ab initio potential, in comparison with experimental data (Refs. 19 and 22-24) (lower numbers). The energies are drawn to scale (using experimental values), except for the small interchange splittings which are enlarged by a factor of 10 .

$$
\begin{aligned}
B_{\mathrm{QM}}^{(1)}(T)= & B_{\mathrm{cl}}(T)+\frac{\hbar^{2}}{24\left(k_{B} T\right)^{3}} \\
& \times \int d \boldsymbol{R}\left\langle e^{-V / k_{B} T} \frac{1}{2} \sum_{i=1}^{2}\left[\frac{\boldsymbol{F}_{i}^{2}}{M}+\sum_{\alpha} \frac{\tau_{\alpha i}^{2}}{I_{\alpha}}\right]\right\rangle_{\Omega_{1} \Omega_{2}},
\end{aligned}
$$

where the notation $B_{\mathrm{OM}}^{(1)}$ emphasizes that this quantity contains the first-order "(1)" quantum-mechanical (QM) correction to the classical value $B_{\mathrm{cl}}(T)$ defined as

$$
B_{\mathrm{cl}}(T)=-\frac{1}{2} \int d \boldsymbol{R}\left\langle e^{-V / k_{B} T}-1\right\rangle_{\Omega_{1} \Omega_{2}} .
$$

In the equations above, $k_{B}$ is the Boltzmann constant, $\boldsymbol{R}$ is the vector connecting the centers of mass of the two monomers, $\Omega_{1}$ and $\Omega_{2}$ are two sets of Euler angles (in a laboratory frame) describing the orientations of the two monomers, and the shorthand notation \langle\rangle$_{\Omega_{1} \Omega_{2}} \equiv\left[1 /\left(8 \pi^{2}\right)^{2}\right] \int d \Omega_{1} d \Omega_{2}$ is used for angular averaging. The interaction potential $V$ depends on the coordinates $\left(\boldsymbol{R}, \Omega_{1}, \Omega_{2}\right), \boldsymbol{F}_{i}, i=1,2$ is the force on molecule $i$ exerted by the partner, and $\tau_{\alpha i}, i=1,2, \alpha=1,2,3$, are the components of the torque on molecule $i$ along the principal axis $\alpha$ of this molecule. The mass of the molecule and its principal moments of inertia are denoted by $M$ and $I_{\alpha}$, 


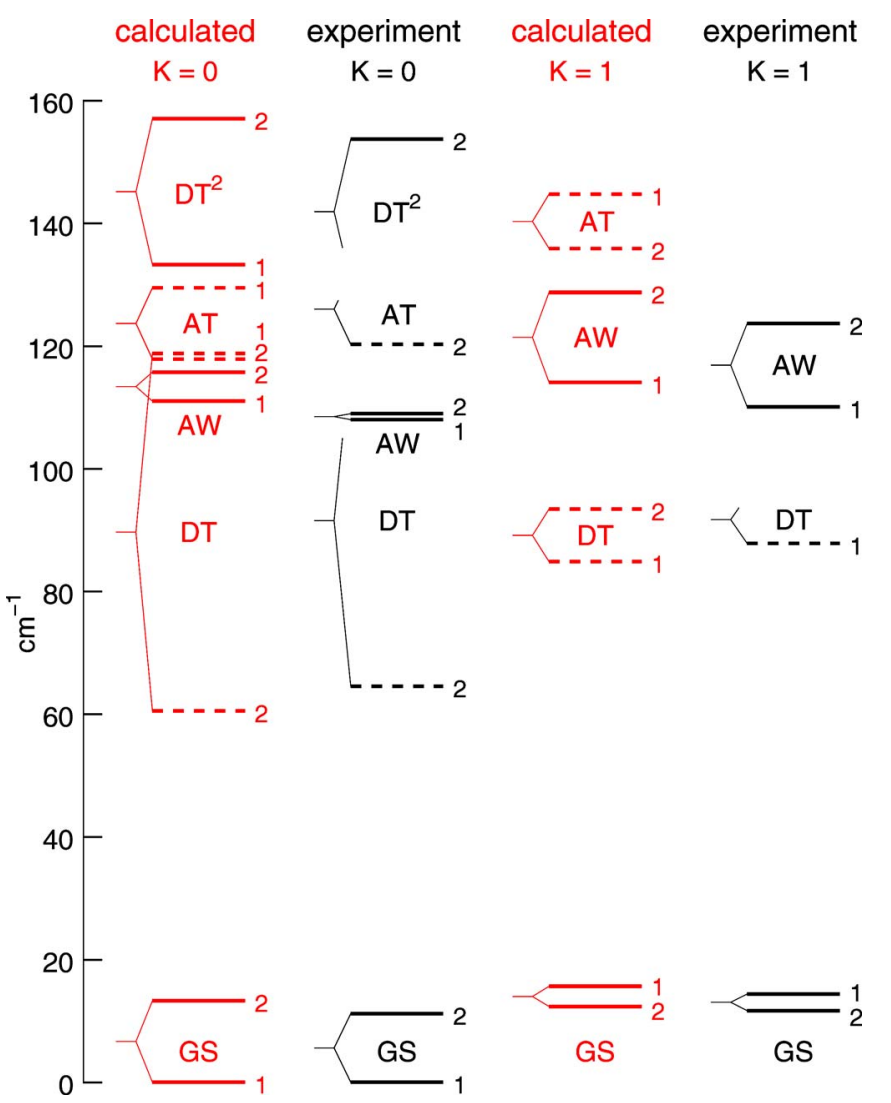

FIG. 3. (Color online) VRT levels of the $\mathrm{H}_{2} \mathrm{O}$ dimer corresponding to the intermolecular vibrations, calculated from the $\mathrm{CC}$-pol potential, in comparison with experimental data (Refs. 25 and 66). The levels 1 and 2 are the origins, $o_{1}$ and $o_{2}$, of the $A_{1}, E_{1}, B_{1}$ and $A_{2}, E_{2}, B_{2}$ levels, respectively. The abbreviations GS, DT, AW, and AT denote the ground state $\left(A^{\prime}\right)$, donor torsion $\left(A^{\prime \prime}\right)$, acceptor wag $\left(A^{\prime}\right)$, and acceptor twist $\left(A^{\prime \prime}\right)$ modes, following Refs. 25 and 27. Solid lines refer to $A^{\prime}$ symmetry, dashed lines to $A^{\prime \prime}$ symmetry, with respect to the point group $C_{s}$ of the equilibrium structure. Experimental levels not shown have not been measured to date.

$\alpha=1,2,3$, respectively. The radial parts of the integrals in Eqs. (3) and (4) are readily simplified to $4 \pi \int_{0}^{\infty} R^{2} d R$ with the integrands (angular averages) evaluated for some fixed vector $\boldsymbol{R}$, e.g., pointing along the $z$ axis. Since for a fixed $\boldsymbol{R}$ the potential $V$ depends only on 5 out of 6 monomer angular coordinates, the integrals in Eqs. (3) and (4) are six dimensional.

While the approximation of Eq. (3) works well for intermediate and high temperatures, higher-order quantum effects are expected to become important as the temperature decreases. These effects have been investigated by Schenter, ${ }^{72}$ who calculated the exact quantum-mechanical second virial coefficient for water using the path integral technique with several available potentials. One of the conclusions from Ref. 72 was that the values of $B(T)$ obtained in the converged path integral simulations could be reproduced with the accuracy of a few percent using the classical expression (4) with the potential $V$ replaced by the temperaturedependent effective potential

$$
V_{\mathrm{eff}}=V+\frac{\hbar^{2}}{24\left(k_{B} T\right)^{2}} \sum_{i=1}^{2}\left[\frac{\boldsymbol{F}_{i}^{2}}{M}+\sum_{\alpha} \frac{\tau_{\alpha i}^{2}}{I_{\alpha}}\right] .
$$

Following Schenter, ${ }^{72}$ the approximation to $B(T)$ obtained in this way will be denoted by $B_{\mathrm{QM}}^{\mathrm{TI}}$ to acknowledge the work of

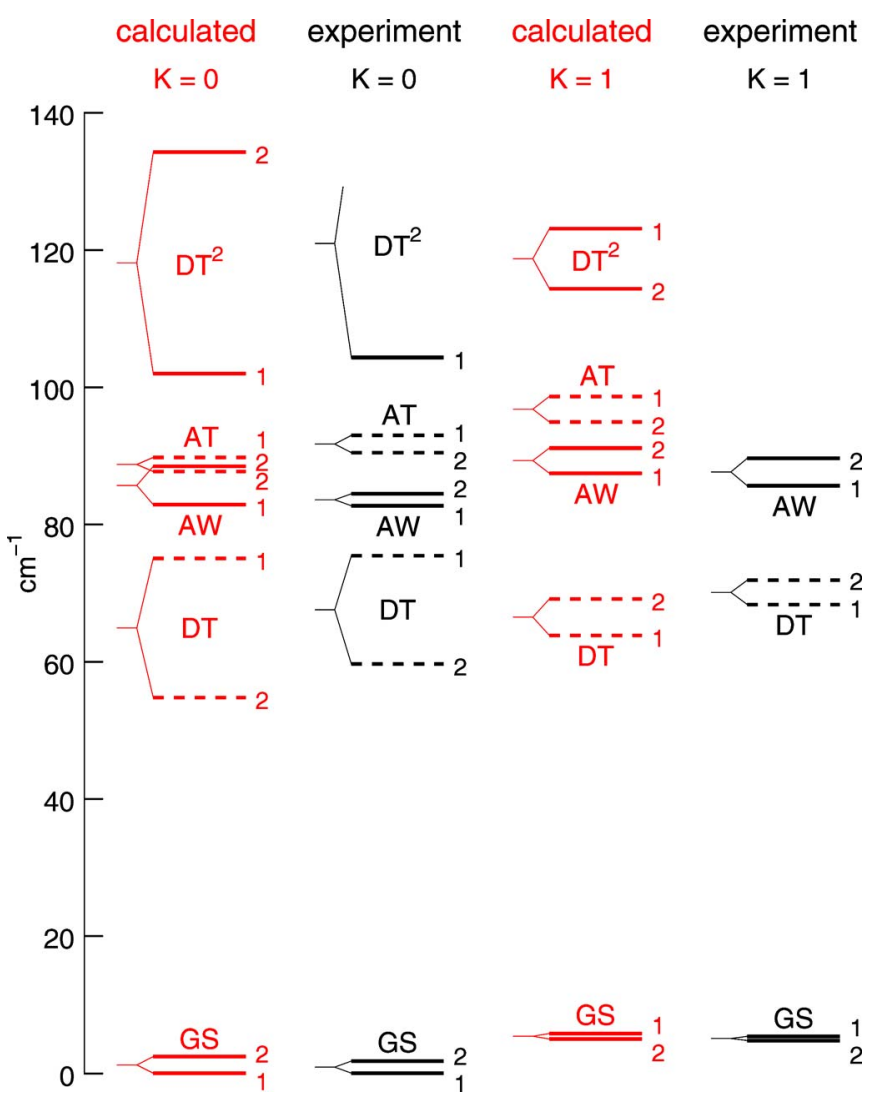

FIG. 4. (Color online) VRT levels of the $\mathrm{D}_{2} \mathrm{O}$ dimer corresponding to the intermolecular vibrations, calculated from the CC-pol potential, in comparison with experimental data (Refs. 24 and 66). All symbols are explained in Fig. 3, except for $\mathrm{DT}^{2}$ which denotes the donor torsion overtone $\left(A^{\prime}\right)$ (Ref. 27). In Ref. 25 this mode was referred to as in-plane bend (IB).

Takahashi and Imada, ${ }^{73}$ who introduced the concept of an effective potential for improving numerical schemes of path integration. The quantity $B_{\mathrm{QM}}^{\mathrm{TI}}$ offers an accuracy comparable to the exact quantum calculation at the same low cost as

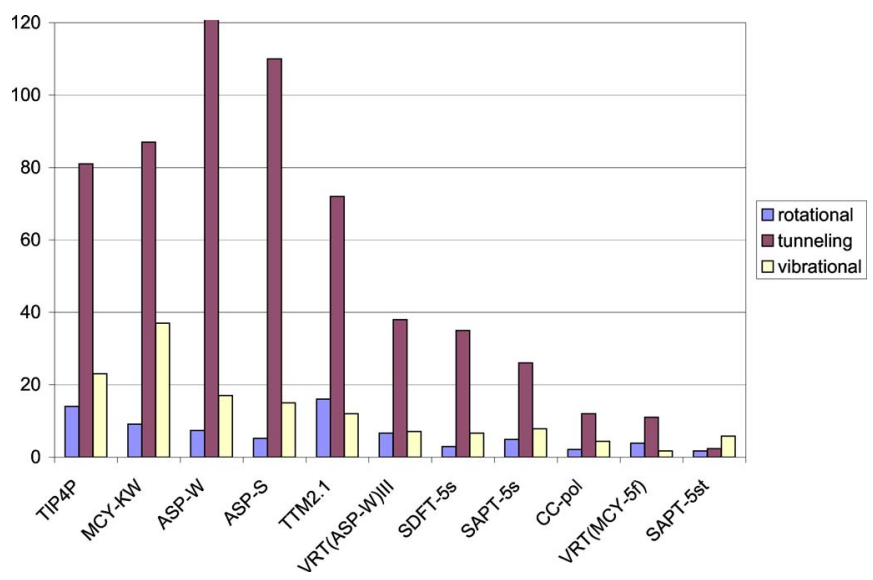

FIG. 5. (Color online) Root mean square relative percentage deviations from experiments for various parts of the $\mathrm{H}_{2} \mathrm{O}$ dimer spectra. The "rotational" deviations include $A$ and $B+C$ rotational constants for the ground state. The "tunneling" deviations include the ground state $a(0)+a(1), i_{1}$, and $i_{2}$ splittings. The "vibrational" deviations include the frequencies of the intermolecular vibrations DT, AW, AT, and $\mathrm{DT}^{2}$ for $K=0$. See previous figures for spectral notation. For sources of the potentials and VRT calculations see Refs. 8, 11, 13, 28, 30, 34, 35, 39, 42-45, and 67. The error of the tunneling splittings from the ASP-W potential is $325 \%$. 
TABLE III. Second virial coefficient of water in $\mathrm{cm}^{3} / \mathrm{mol}$. See text for explanations of symbols. Numerical integration errors are below $0.5 \%$ for $T<1000 \mathrm{~K}$ and below $0.3 \mathrm{~cm}^{3} / \mathrm{mol}$ for $T>1000 \mathrm{~K}$.

\begin{tabular}{|c|c|c|c|c|c|c|c|}
\hline \multirow[b]{2}{*}{$T / \mathrm{K}$} & \multicolumn{3}{|c|}{ SAPT-5s } & \multicolumn{3}{|c|}{ CC-pol } & \multirow[b]{2}{*}{ Expt. ${ }^{a}$} \\
\hline & $B_{\mathrm{cl}}(T)$ & $B_{\mathrm{QM}}^{(1)}$ & $B_{\mathrm{QM}}^{\mathrm{TI}}$ & $B_{\mathrm{cl}}(T)$ & $B_{\mathrm{QM}}^{(1)}$ & $B_{\mathrm{QM}}^{\mathrm{TI}}$ & \\
\hline 273.15 & -2031.9 & -1407.6 & -1550.4 & -2360.8 & -1377.2 & -1671.2 & -1916.9 \\
\hline 293.15 & -1384.3 & -1029.6 & -1100.0 & -1572.0 & -1028.9 & -1172.2 & -1307.8 \\
\hline 295.15 & -1336.7 & -1000.2 & -1066.0 & -1514.9 & -1000.8 & -1134.8 & -1262.9 \\
\hline 298.15 & -1269.7 & -958.2 & -1017.9 & -1434.6 & -960.6 & -1081.9 & -1199.7 \\
\hline 323.15 & -863.7 & -690.8 & -718.6 & -954.4 & -698.8 & -755.0 & -816.7 \\
\hline 373.15 & -474.8 & -407.6 & -415.2 & -507.8 & -413.0 & -428.6 & -451.6 \\
\hline 423.15 & -302.3 & -270.0 & -272.6 & -316.2 & -272.1 & -277.7 & -289.9 \\
\hline 448.15 & -250.1 & -226.4 & -228.0 & -259.4 & -227.4 & -231.0 & -240.6 \\
\hline 473.15 & -210.7 & -192.7 & -193.7 & -216.9 & -192.9 & -195.3 & -203.2 \\
\hline 523.15 & -155.6 & -144.6 & -145.0 & -158.3 & -143.9 & -145.0 & -150.5 \\
\hline 573.15 & -119.7 & -112.4 & -112.5 & -120.5 & -111.1 & -111.8 & -115.7 \\
\hline 673.15 & -76.4 & -72.7 & -72.6 & -75.8 & -71.1 & -71.3 & -73.6 \\
\hline 773.15 & -51.8 & -49.6 & -49.6 & -50.8 & -48.1 & -48.2 & -49.7 \\
\hline 873.15 & -36.3 & -34.9 & -34.8 & -35.2 & -33.4 & -33.5 & -34.6 \\
\hline 973.15 & -25.7 & -24.7 & -24.7 & -24.6 & -23.4 & -23.4 & -24.4 \\
\hline 1000 & -23.4 & -22.5 & -22.5 & -22.4 & -21.3 & -21.3 & -22.2 \\
\hline 1100 & -16.4 & -15.7 & -15.7 & -15.4 & -14.6 & -14.6 & -15.5 \\
\hline 1200 & -11.1 & -10.6 & -10.6 & -10.2 & -9.6 & -9.6 & -10.4 \\
\hline 1300 & -7.0 & -6.6 & -6.6 & -6.2 & -5.7 & -5.7 & -6.5 \\
\hline 1400 & -3.7 & -3.4 & -3.4 & -3.0 & -2.7 & -2.7 & -3.4 \\
\hline 1500 & -1.1 & -0.8 & -0.8 & -0.5 & -0.2 & -0.2 & -0.8 \\
\hline 1600 & 1.1 & 1.3 & 1.3 & 1.6 & 1.9 & 1.9 & 1.3 \\
\hline 1800 & 4.4 & 4.6 & 4.6 & 4.8 & 5.0 & 5.0 & 4.5 \\
\hline 2000 & 6.8 & 7.0 & 6.9 & 7.1 & 7.2 & 7.2 & 6.9 \\
\hline 2500 & 10.5 & 10.8 & 10.6 & 10.6 & 10.7 & 10.7 & 10.6 \\
\hline 3000 & 12.5 & 12.9 & 12.6 & 12.6 & 12.6 & 12.6 & 12.6 \\
\hline
\end{tabular}

${ }^{\mathrm{a}}$ Reference 29.

required by $B_{\mathrm{QM}}^{(1)}$ of Eq. (3). It is easily seen that both these approximations are consistent up to the order of $\hbar^{2}$. Recently, Bustos Marún et al. ${ }^{74}$ used the $B_{\mathrm{QM}}^{\mathrm{TI}}$ approach to compute the quantum second virial coefficients for a number of water potentials, including the VRT(ASP-W) family ${ }^{41,42}$ and SAPT-5st. ${ }^{28}$

The six-dimensional integrals involved in calculations of $B(T)$ were performed as described in Ref. 30, using a Monte Carlo technique with two million configurations for angular coordinates and a numerical quadrature for the radial coordinate. The numerical accuracy of the integration is better than $0.5 \%$ for temperatures below $1000 \mathrm{~K}$ and better than $0.3 \mathrm{~cm}^{3} / \mathrm{mol}$ for higher temperatures. The results of the calculations with the CC-pol potential are presented in Table III and Fig. 6 and compared to their counterparts obtained previously with the SAPT-5s potential, ${ }^{30}$ as well as to the results from the recent correlation of experimental data compiled by Harvey and Lemmon. ${ }^{29}$ The total $B(T)$ 's computed with the CC-pol potential have been presented in a figure in Ref. 13 . The $B_{\mathrm{QM}}^{\mathrm{TI}}(T)$ values corresponding to the SAPT-5s potential have been calculated in the present work.

It is clear from Table III that the classical approximation produces results which are too negative. For low temperatures, the $B_{\mathrm{cl}}(T)$ values obtained from the CC-pol potential are more negative than the SAPT-5s ones, which is consistent with the well of the former potential being deeper. The inclusion of the first quantum correction in $B_{\mathrm{QM}}^{(1)}$ changes the sign of the error and actually causes the SAPT-5s results to deviate even more from experiment in terms of absolute value. On the other hand, the CC-pol potential produces $B_{\mathrm{OM}}^{(1)}$ at approximately the same distance from experiment as its classical counterpart. The role of the higher-order quantum corrections is clearly visible at low temperatures. These effects provide a negative contribution, thus bringing the results from both potentials to a closer agreement with experiment. The difference between $B_{\mathrm{QM}}^{(1)}$ and $B_{\mathrm{QM}}^{\mathrm{TI}}$ vanishes quickly with increasing temperature, but the total quantum effect is still visible for temperatures well above $1000 \mathrm{~K}$. The magnitudes of the quantum correction to $B(T)$ (i.e., the differences between the quantum and classical values), both at the firstorder and the "TI" level, are larger for the CC-pol potential than for SAPT-5s, which is especially visible for low temperatures. Since the quantum corrections depend on the derivatives of the potential, these derivatives must be larger on average in the case of CC-pol. The larger quantum corrections for CC-pol result in the TI level values from CC-pol and SAPT-5s being closer to each other than in the case of classical ones. The agreement with experiment will be discussed below.

An experimentally measured quantity resulting exclusively from quantum effects is the so-called isotope shift, i.e., the change of the second virial coefficient upon replacement of hydrogen atoms of both molecules with deuterium. Table IV compares the isotope shifts computed at the TI 


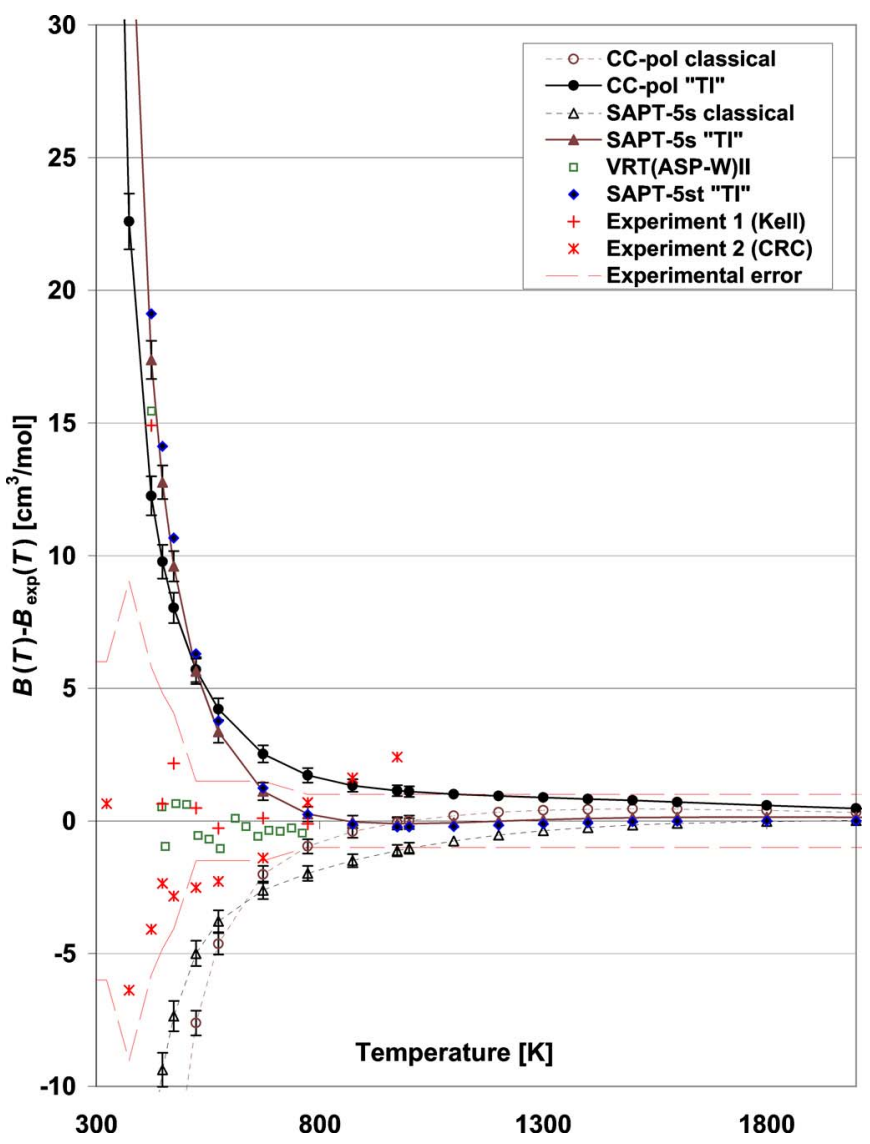

FIG. 6. (Color online) Second virial coefficient of steam relative to the results of correlation of experimental values performed by Harvey and Lemmon (Ref. 29). Data labeled "Experiment 1" and "Experiment 2" are from Refs. 75 and 76, respectively. Error bars on CC-pol and SAPT-5s data show only the accuracy of numerical integration; the errors of SAPT-5st points are similar. For $T>1000 \mathrm{~K}$ the error bars are smaller than the size of symbols. The VRT(ASP-W)II results are from Ref. 42 and were computed at the $B_{\mathrm{QM}}^{(1)}$ level.

level with CC-pol and SAPT-5s potentials to the available experimental ones. Data computed in Ref. 77 from the original MCY ab initio potential ${ }^{46}$ are also shown. Despite differences in the quantum corrections themselves, the CC-pol and SAPT-5s potentials give very similar isotope shifts, which agree with experiment quite well for lower temperatures, but fall outside of experimental error bars as the temperature gets higher. The MCY values, on the other hand, differ from ex-

TABLE IV. Isotope shift of the second virial coefficient of water (in $\left.\mathrm{cm}^{3} / \mathrm{mol}\right)$ calculated as the difference between $B_{\mathrm{QM}}^{\mathrm{TI}}(T)$ of $\mathrm{H}_{2} \mathrm{O}$ and that of $\mathrm{D}_{2} \mathrm{O}$. The uncertainties of theoretical values are sums of numerical integration errors.

\begin{tabular}{ccrrc}
\hline \hline Temperature (K) & Experiment $^{\mathrm{a}}$ & SAPT-5s & CC-pol & MCY $^{\mathrm{b}}$ \\
\hline 423.15 & $15 \pm 7$ & $12.6 \pm 1.5$ & $15.6 \pm 1.5$ & 46.5 \\
448.15 & $10 \pm 5$ & $9.5 \pm 1.3$ & $11.6 \pm 1.3$ & 35.3 \\
473.15 & $3.9 \pm 1$ & $7.3 \pm 1.1$ & $8.9 \pm 1.1$ & 27.6 \\
523.15 & $1.8 \pm 0.3$ & $4.6 \pm 1.0$ & $5.5 \pm 0.9$ & 18.3 \\
573.15 & $0.5 \pm 0.4$ & $3.1 \pm 0.8$ & $3.7 \pm 0.8$ & 13.1 \\
673.15 & $0.6 \pm 0.3$ & $1.6 \pm 0.7$ & $1.9 \pm 0.6$ & 8.01 \\
773.15 & $0.0 \pm 0.2$ & $1.0 \pm 0.6$ & $1.1 \pm 0.5$ & 5.66 \\
\hline \hline
\end{tabular}

${ }^{\mathrm{a}}$ Reference 75 .

${ }^{\mathrm{b}}$ Reference 77 , calculation at the $B^{(1)}(T)$ level. periment by a factor of 3-4 for low $T$ (and much more for higher $T$ ), showing that accuracy of the potential is crucial for reproducing the isotope shifts.

Figure 6 presents the differences between the theoretical $B(T)$ calculated from the CC-pol and SAPT-5s potentials with and without quantum corrections and the recent experimental correlation of Ref. 29 (the horizontal axis). We use here a difference plot instead of plotting $B(T)$ straightforwardly (as in Fig. 2 of Ref. 13) to better distinguish several sets of data that are compared. Results obtained in Ref. 42 with the VRT(ASP-W)II empirical pair potential at the $B_{\mathrm{QM}}^{(1)}$ level are also shown, as well as two other sets of experimental values. It is clear that the results of Kell et al..$^{75}$ are overall in better agreement with the correlation of Harvey and Lemmon $^{29}$ than the CRC compilation. ${ }^{76}$ The former results are also generally closer to the theoretical values with quantum effects included. At low temperatures, the errors of the $B_{\mathrm{OM}}^{\mathrm{TI}}$ results from the CC-pol potential are smaller than those given by the SAPT-5s potential, but for $T>500 \mathrm{~K}$ the latter results become closer to experiment. As expected, at the high-temperature end, both the CC-pol and SAPT-5s quantum curves converge to their respective classical curves. The CC-pol results are almost $1 \mathrm{~cm}^{3} / \mathrm{mol}$ above the experiment for $T=1000 \mathrm{~K}$. On the other hand, the SAPT-5s difference curve seems to be asymptotically approaching zero (see a further discussion below). The VRT(ASP-W)II results provide the best overall agreement with experiment (the small oscillations around the horizontal axis are more likely a result of inaccurate digitization of Fig. 6 in Ref. 42 than a real physical effect), although the value at $T=423.15 \mathrm{~K}$ has an error larger than that of the CC-pol result. However, the VRT(ASP-W)II values were computed only at the $B_{\mathrm{QM}}^{(1)}(T)$ level. The inclusion of higher-order quantum corrections would shift these values down by about $2-6 \mathrm{~cm}^{3} / \mathrm{mol}$ in the range of $400-500 \mathrm{~K}$ and $1-2 \mathrm{~cm}^{3} / \mathrm{mol}$ in the range of $500-600 \mathrm{~K}$. The virial coefficients produced by another potential tuned to experimental VRT data, SAPT- 5 st ${ }^{28}$ closely follow the results from its ab initio predecessor, SAPT-5s (during the course of this work it was found that the SAPT5st data presented in Fig. 8 of Ref. 30, suggesting that the latter potential gives $B(T)$ significantly less negative than SAPT-5s, were incorrect). To our knowledge, the virial coefficients have not been published for the TTM2.1 potential $^{39}$ and are available only for the TTM (Ref. 78) and TTM2-R (Ref. 37) potentials and only in a relatively narrow range of temperatures between 423 and $773 \mathrm{~K}$. The TTM coefficients are significantly too negative relative to experiment. The TTM2-R coefficients, computed at the $B_{\mathrm{QM}}^{(1)}(T)$ level, are above experiment and very close to SAPT-5s values at the lower end of the range, but become too negative at the upper end (lie $2.1 \mathrm{~cm}^{3} / \mathrm{mol}$ below the lowest point marked on Fig. 6 for $T=773.15 \mathrm{~K}$ ).

For temperatures below about $700 \mathrm{~K}$, both the SAPT-5s and CC-pol results fall above the error envelope of the experimental correlation proposed by Harvey and Lemmon. ${ }^{29}$ This observation, seemingly disappointing for CC-pol, should be considered with caution. In Ref. 74, Bustos Marún et al. suggested that monomer flexibility effects, neglected in all theoretical calculations described here, could have a large 
effect on the second virial coefficients around room temperature. In an upcoming work, ${ }^{79}$ we show that such effects are indeed sizable and negative, which would bring both the CCpol and SAPT-5s results closer to experiment, while the VRT(ASP-W)II results would fall further below the experimental ones. In the high-temperature range, the virial results from all potentials considered are within the experimental error bars, estimated at about $1 \mathrm{~cm}^{3} / \mathrm{mol}$, with the SAPT-5s potential giving the best agreement with experiment. However, in view of the scarcity of actual experimental results in this region, the correlation of Harvey and Lemmon ${ }^{29}$ treated the SAPT-5s results as a substitute for high- $T$ experimental data points. The magnitude of $B(T)$ at high temperatures [especially close to the Boyle temperature of 1538 K (Ref. 29), i.e., when $B(T)=0$ ] is determined by a subtle balance between the positive contributions of the repulsive wall of the potential and the negative contribution of the attractive well including the large- $R$ asymptotic region. Since both these regions are described more accurately by $\mathrm{CC}$-pol than by SAPT-5s, we believe that the $B_{\mathrm{QM}}^{\mathrm{TI}}(T)$ values given by the former potential are the best representation of the rigidmonomer second virial coefficient of water, including the high-temperature region. The remaining deviations from the true second virial coefficients are likely to be due to monomer flexibility effects.

\section{LIQUID-PHASE SIMULATIONS}

Even the most accurate pair interaction potential alone is not sufficient to properly describe larger water clusters and liquid or solid-state water, where many-body effects play an important role. Until recently, nonadditive effects other than those resulting from induction interactions have almost always been neglected in simulations of water. In Ref. 48, a complete three-body nonadditive potential was developed containing, besides the induction component, also the shortrange exchange nonadditive terms. The latter potential has been used, ${ }^{2}$ along with the SAPT- 5 s pair potential, ${ }^{30}$ to investigate the convergence of the many-body expansion for liquid water. One of the conclusions of that work was that indeed, the induction nonadditivity is dominant in liquid water and that other, short-range three-body terms play a lesser role in the determination of its structure and energetics. Also, the role of the nonadditive effects higher than the three-body ones was found to be very small. However, the results of the simulations of Ref. 2 were not fully convincing, as the potential used led to relatively significant discrepancies with experiment in reproducing the tetrahedral structure of the liquid. As we know now, ${ }^{13}$ the main source of the discrepancies was the residual inaccuracy of SAPT-5s. In this section, we will reexamine the convergence of the many-body expansion for water using the CC-pol potential.

\section{A. Many-body potential}

To evaluate the roles of different nonadditive terms, we followed Ref. 2 and considered several variants of the $\mathrm{N}$-body interaction potential, containing nonadditive interac- tions at different levels. The simplest of these variants is the pure two-body potential, which, for $N$ molecules in a simulation box, can be calculated as

$$
U_{2 \mathrm{~B}}=U_{0}+\sum_{A<B}^{N} V_{2}^{\text {ind }}(A, B),
$$

where $A$ and $B$ stand for the positions and orientations of molecules. The component $U_{0}$ is the sum of the "noninduction" parts of the CC-pol pair potential of Refs. 13 and 18, i.e.,

$$
U_{0}=\sum_{A<B}^{N} \sum_{a \in A, b \in B} u_{a b}\left(r_{a b}\right),
$$

where the individual site-site "potentials" $u_{a b}\left(r_{a b}\right)$, depending on the distances $r_{a b}$ between two sites located on different monomers, have the form ${ }^{18}$

$$
\begin{aligned}
u_{a b}\left(r_{a b}\right)= & \left(1+\sum_{m=1}^{3} a_{m}^{a b} r_{a b}^{m}\right) e^{\alpha_{a b}-\beta_{a b} r_{a b}}+f_{1}\left(\delta_{1}^{a b}, r_{a b}\right) \frac{q_{a} q_{b}}{r_{a b}} \\
& +\sum_{n=6,8,10} f_{n}\left(\delta_{n}^{a b}, r_{a b}\right) \frac{C_{n}^{a b}}{r_{a b}^{n}} .
\end{aligned}
$$

The functions $u_{a b}$ contain an exponential term modeling the exchange and overlap effects, an electrostatic term with site charges $q_{a}, q_{b}$, and $r_{a b}^{-n}$ terms with coefficients $C_{n}^{a b}$ responsible mainly for the asymptotic part of the dispersion interaction. All the inverse power terms are multiplied by the Tang-Toennies damping functions ${ }^{80}$

$$
f_{n}(\delta, r)=1-e^{-\delta r} \sum_{m=0}^{n} \frac{(\delta r)^{m}}{m !} .
$$

The term $V_{2}^{\text {ind }}(A, B)$ in Eq. (6) approximates the two-body induction component of the interaction energy. It is a special case of the $N$-body induction energy in the asymptotic polarization model given by

$$
V_{N}^{\text {ind }}=-\frac{1}{2} \sum_{i=1}^{N} \boldsymbol{E}_{i} \cdot \boldsymbol{\mu}_{i}^{\text {ind }},
$$

where it is understood that $V_{N}^{\text {ind }}$ depends on the positions and orientations of all molecules, $\boldsymbol{E}_{i}$ is the static electrostatic field on the polarizable center of molecule $i$, and $\boldsymbol{\mu}_{i}^{\text {ind }}$ is the dipole moment induced on molecule $i$. For detailed definitions of these quantities and procedures of their evaluation; see Refs. 2, 18, and 48. Note that for just a single dimer AB, the pair potential is

$$
V=\sum_{a \in A, b \in B} u_{a b}\left(r_{a b}\right)+V_{2}^{\text {ind }}(A, B) .
$$

Simulation results obtained with the pairwise-additive potential $U_{2 \mathrm{~B}}$ of Eq. (6) will be referred to as "CC-pol."

The next step is to include the three-body nonadditive interactions, described by the $3 \mathrm{~B}$ (SAPT) potential of Ref. 48 . Denoting this potential, calculated for the trimer $A B C$, by 
$v_{3}(A, B, C)$, we obtain

$$
U_{3 \mathrm{~B}}=U_{2 \mathrm{~B}}+\sum_{A<B<C}^{N} v_{3}(A, B, C)
$$

as the potential for a system of $N$ molecules. The last term on the right hand side of the equation accounts for all threebody effects contained at the HF level of theory, ${ }^{48}$ including the three-body induction as well as some short-range nonadditive effects of exchange nature (i.e., resulting from Pauli's exclusion principle). Simulations performed with the $U_{3 \mathrm{~B}}$ potential will be referred to as "CC-pol+3B."

Extension of the $U_{3 \mathrm{~B}}$ potential to include higher-body nonadditive effects is not straightforward, especially if shortrange components of such effects are of interest. However, the $N>$ three-body induction energy can be estimated relatively easily by using the polarization term $V_{N}^{\text {ind }}$. One has to realize, however, that the three-body induction component is already included in the potential $v_{3}$ of Ref. 48 and therefore this component must be removed from $V_{N}^{\text {ind }}$ to avoid double counting. The "complete" $N$-body potential then becomes

$$
\begin{aligned}
U_{\mathrm{NB}}= & U_{0}+\sum_{A<B<C}^{N} v_{3}(A, B, C)+V_{N}^{\text {ind }} \\
& -\sum_{A<B<C}^{N}\left[V_{3}^{\text {ind }}(A, B, C)-V_{2}^{\text {ind }}(A, B)-V_{2}^{\text {ind }}(B, C)\right. \\
& \left.-V_{2}^{\text {ind }}(A, C)\right],
\end{aligned}
$$

where the last sum over trimers is the three-body nonadditive part of $V_{N}^{\text {ind }}$. Note that $V_{N}^{\text {ind }}$ contains also the two-body induction term, which complements $U_{0}$ in reproducing the pairwise-additive component of the interaction energy. Simulations performed in this way will be denoted "CC-pol +NB." We should point out that the three-body potential $v_{3}(A, B, C)$ was used in exactly the same form as in Ref. 48 . Thus, the monomer permanent charges and the polarizability in $v_{3}(A, B, C)$ are slightly different from those applied in CC-pol.

Finally, it is interesting to test the outcome of neglecting the short-range nonadditive effects and leaving only the many-body asymptotic induction term in the interaction potential. Such an approximation, which amounts to using

$$
U_{\mathrm{NB}(\text { ind })}=U_{0}+V_{N}^{\text {ind }}
$$

as the $N$-body interaction potential, is the most straightforward way of utilizing the polarizable character of CC-pol. Results of simulations of this kind will be denoted by "CC-pol+NB(ind)."

\section{B. Details of molecular dynamics simulations}

Our molecular dynamics simulations were performed in the NVE ensemble (constant number of molecules, volume, and energy) at a density of $1 \mathrm{~g} / \mathrm{cm}^{3}$ with 128,256 , or 512 molecules in a cubic periodic box with edge size $L$ equal to $15.64,19.71$, and $24.83 \AA$, respectively. Starting from a simple cubic lattice with random molecular orientations, the system was first equilibrated for 5 ps using the $U_{2 \mathrm{~B}}$ potential, during which time the velocities were scaled to the target temperature of $298.15 \mathrm{~K}$. A similar equilibration with velocity scaling was then performed with the potential of interest. After a subsequent 5 ps equilibration in the NVE ensemble, averages were collected for an additional 10-20 ps. The time step was equal to $1 \mathrm{fs}$ and the Verlet algorithm in the position representation was used to integrate the classical equations of motion. The atomic positions were propagated in Cartesian coordinates and constraints were imposed on three interatomic distances within each molecule to keep the molecules rigid. The electrostatic terms in Eq. (8) were split as

$$
f_{1} \frac{q_{a} q_{b}}{r_{a b}}=\frac{q_{a} q_{b}}{r_{a b}}+\left(f_{1}-1\right) \frac{q_{a} q_{b}}{r_{a b}} .
$$

The first term on the right-hand side contains long-range components and has been evaluated using Ewald summation, while the second term vanishes exponentially and has been summed in real space, together with the other short-range terms of the potential.

To avoid a total energy drift in the MD simulations, all parts of the interaction potential (except for the long-range electrostatics treated with the Ewald summation) were smoothly turned off near the cutoff distance by multiplication with the factor

$$
f_{\mathrm{lr}}(r)=\left(1+e^{\gamma\left(r-r_{\mathrm{c}}\right)}\right)^{-\delta},
$$

where $r$ is the distance between the centers of mass of the two molecules, $r_{\mathrm{c}}$ is the cutoff distance (taken as half of the simulation box length), $\gamma=10 \AA^{-1}$, and $\delta=33.22$ has been chosen so that $f_{\mathrm{lr}}\left(r_{\mathrm{c}}\right)=10^{-10}$. The factor, which rapidly changes from 1 to 0 near $r_{\mathrm{c}}$, was applied for all $r$. In the case of the iterated induction terms $V_{n}^{\text {ind }}, n=2,3, N$, the factor $f_{\text {lr }}$ was used to damp the total electric field (from both the static charges and the induced dipole) of one molecule on the polarizable center of the other. More precisely, $\boldsymbol{T}_{i j}$ in Eq. (12) of Ref. 18 was replaced by $f_{\mathrm{lr}}\left(r_{i j}\right) \boldsymbol{T}_{i j}$ and the static electric field $\boldsymbol{E}_{i}$ in Eq. (10) and in Eq. (12) of Ref. 18 was computed as

$$
\boldsymbol{E}_{i}=\sum_{j \neq i} f_{\mathrm{lr}}\left(r_{i j}\right) \boldsymbol{E}_{j i}
$$

where $\mathbf{E}_{j i}$ is the static field generated by charges on molecule $j$ on the polarizable center of molecule $i$.

\section{Many-body convergence of liquid structure}

In Fig. 7, we plot the atom-atom radial distribution functions (RDFs) resulting from the simulations with the CC-pol potential. These RDFs have been presented in Ref. 13, however, here we compare to different experimental data. These data are still the ones published by Soper, ${ }^{51}$ but the curves plotted in Fig. 7 utilize the values tabulated for $T=298 \mathrm{~K}$ on the web page given in Ref. 51. For each RDF, there are two curves listed, cases (a) and (b), corresponding to the two different neutron experiments. The error bars are also taken from the web site. In our previous work, ${ }^{12,13,35}$ we were comparing to the curves from Fig. 6 in Ref. 51 (digitized by us) not realizing that these curves are somewhat different from 

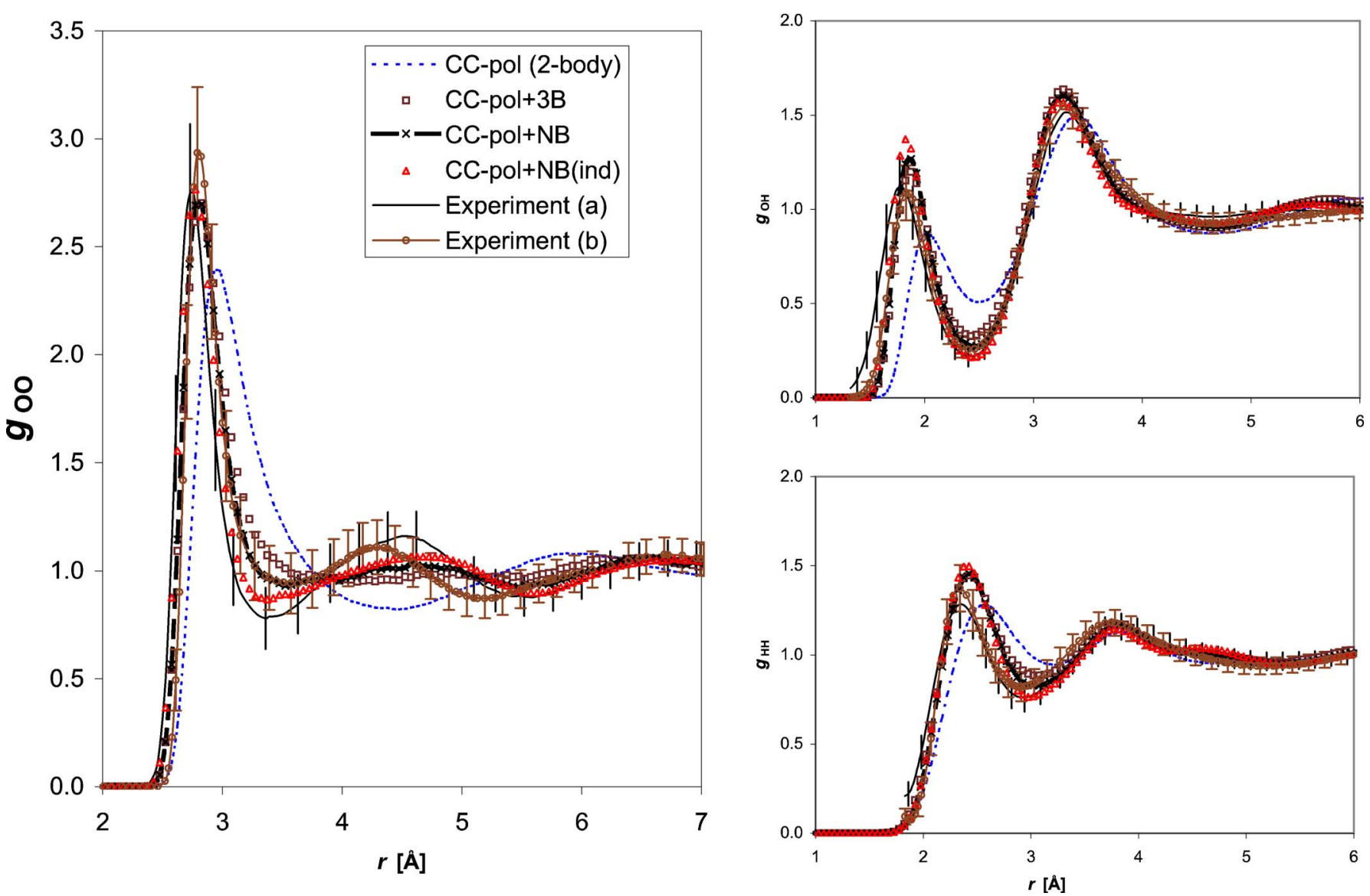

FIG. 7. (Color online) Atom-atom radial distribution functions from simulations based on the CC-pol potential. See text for explanations of acronyms. The experimental curves are from Ref. 51.

the tabulated ones. In particular, cases (a) and (b) are nearly identical in Fig. 6 in Ref. 51. As seen in our Fig. 7, case (a) curves are sufficiently distinct from case (b) to make a difference in an evaluation of theoretical results. However, the two sets of RDFs are within the error bars of one another. One should also point out that the RDFs are not measured directly but are obtained from a complicated processing of raw data involving use of empirical potentials which to some extent influences the final RDFs. The $g_{\mathrm{OO}}$ function is also known from $x$-ray diffraction experiments and the latest results $^{52}$ are consistent with the neutron scattering curves of Ref. 51.

Examining first in Fig. 7 the OO RDF obtained from the two-body CC-pol simulation, one concludes that the arrangement of oxygen atoms in liquid water described only by the pair potential resembles that of an atomic liquid. The main peak in the $g_{\mathrm{OO}}$ curve is shifted to the right compared to the experimental peak and the first minimum, characteristic for a local tetrahedral structure, is shifted by about $1 \AA$ to the right. The integration of this function (multiplied by the factor $4 \pi \rho r^{2}$, where $\rho$ is the number density) up to the first minimum produces a coordination number of 11.9 instead of the expected 4.5-6.0 based on the experimental curves (a) and (b), respectively. Likewise, the first peak in the $g_{\mathrm{OH}}$ curve is suppressed, indicating an insufficient (compared to experiment) number of hydrogen bonds.

Inclusion of three-body effects in the CC-pol+3B simu- lation improves the agreement with experiment considerably. In particular, the first peak in the $g_{\mathrm{OH}}$ curve becomes higher and shifts to the left, indicating an increased number of hydrogen bonds. Also the first peak in the $g_{\mathrm{OO}}$ curve undergoes a shift to the left, resulting in a position virtually identical to that of the experimental peak for case (b) and a coordination number of 5.6 very close to experiment. Beyond the first peak, the CC-pol+3B $g_{\mathrm{OO}}$ function decreases much faster than its CC-pol counterpart, and a weak minimum is produced at a smaller distance $r$ than in the case of CC-pol, but still a significantly larger $r$ than those of the experimental minima. The position of this first minimum shifts much further to the left when the $N>$ three-body induction effects are included, i.e., when one uses the CC-pol+NB potential in the simulation. In fact, the first minimum given by this potential is in perfect agreement with the experimental case (b). The position of the first maximum is nearly unchanged compared to the CC-pol+3B simulation. The impact of the $N>$ three-body effects on the $g_{\mathrm{OH}}$ and $g_{\mathrm{HH}}$ functions is small, and these functions seem to be well converged already at the CC-pol+3B level. Overall, the RDFs from the CC-pol+NB simulation resemble the experimental ones of case (b) remarkably closely: they are almost indistinguishable for most $r$ smaller than $4 \AA$. The agreement with case (a) is somewhat worse and is basically the same as the agreement of cases (a) and (b) with one another. A better agreement with case (a) for the $g_{\mathrm{OO}}$ function is achieved when the three-body ex- 


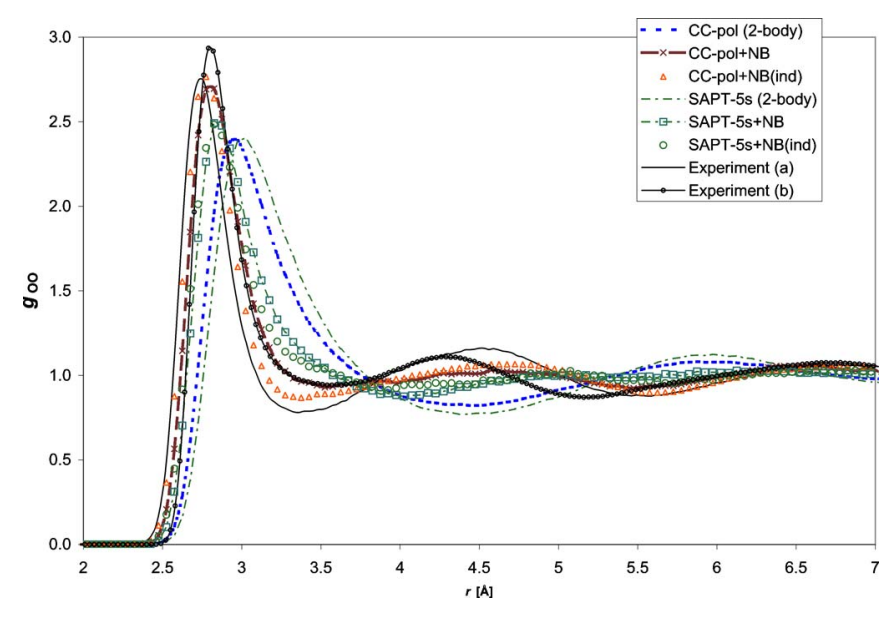

FIG. 8. (Color online) Comparison of the oxygen-oxygen radial distribution functions obtained from simulations based on the CC-pol and SAPT-5s pair potentials. See text for explanations of acronyms. The experimental curve is from Ref. 51.

change interactions are neglected, and only the induction nonadditive contributions is considered, i.e., if the lower level CC-pol+NB(ind) model is used.

The number of hydrogen bonds for a given molecule in the liquid can be estimated from a geometric criterion. ${ }^{81}$ This was done for the SAPT-5s simulations in Ref. 2, but not for the current ones. However, we observed ${ }^{13}$ that there is a good correlation between the coordination number and the number of hydrogen bonds. Using an approximate linear dependence, we found that the $\mathrm{CC}$-pol+NB force field leads to 3.8 hydrogen bonds and CC-pol to 2.7, which quantifies the discussion on the importance of many-body effects. It also indicates that the reports claiming only 2.2 hydrogen bonds, based on x-ray absorption measurements, ${ }^{82,83}$ are not supported by our calculations. These reports have been criticized by several other authors both on experimental ${ }^{84,85}$ and theoretical grounds. ${ }^{86}$

\section{Comparisons to SAPT-5s potential}

It is instructive to compare the liquid structures resulting from our present simulations with those of Ref. 2, where the SAPT-5s pair potential ${ }^{30}$ was used as the two-body component of the interaction energy. Such a comparison is presented in Fig. 8 for the most interesting case of the $g_{\mathrm{OO}}$ function. The results denoted by SAPT-5s and SAPT-5s + NB have been obtained from Monte Carlo simulations analogous to our MD simulations with the CC-pol and CC-pol+NB potentials, respectively (see Ref. 2 for details). Comparing first the two-body SAPT-5s and CC-pol results, one concludes that both pair potentials lead to very similar liquid structures. The peak of the SAPT-5s $g_{\text {Oo }}$ curve is shifted by about $0.05 \AA$ to the right with respect to the CCpol one, consistent with the $\mathrm{O}-\mathrm{O}$ distance in the dimer minimum being larger in the case of SAPT-5s (see Table I in Ref. $35)$. Both two-body curves feature very broad first peaks and broad first minima around $4.5 \AA$, much farther out than the experimental minimum. While augmenting the $\mathrm{CC}$-pol pair potential with nonadditive effects in the $\mathrm{CC}$-pol+NB or $\mathrm{CC}$-pol $+\mathrm{NB}$ (ind) simulations clearly results in the onset of a tetrahedral structure and generally good agreement with ex- periment, the same cannot be said about the SAPT $-5 s+\mathrm{NB}$ simulation. The main peak and the first minimum of the $g_{\mathrm{OO}}$ function from SAPT-5s + NB are still too far to the right and the oxygen coordination number is 8.6. Moreover, as discussed in Ref. 2, the SAPT $-5 \mathrm{~s}+\mathrm{NB}$ curve turns out to be very similar to the SAPT $-5 s+3 B$ one (not shown), which would suggest that the liquid structure is not dependent on $N>$ three-body interactions. Quite a different observation can be made based on Fig. 7 , where the $N>$ three-body induction nonadditivities are the source of a substantial difference between the CC-pol+3B and CC-pol+NB curves.

The overall conclusion from the SAPT-5s and CC-pol sets of simulations is that the liquid structure results from a subtle balance between the two-body and nonadditive portions of the interaction energy. In the absence of the latter, both two-body potentials lead to very similar, unstructured liquids, which show that the preference for hydrogen-bonded configurations at the two-body level is not sufficient for a tetrahedral structure to develop. The nonadditive forces generally tend to bring the molecules closer together by providing extra attraction. However, only the CC-pol pair potential (and the SDFT-5s potential of Ref. 35) has the ability to utilize this attraction to lock the molecules into tetrahedral coordination, which qualitatively changes the structure. It is astonishing that relatively small changes in the force field make the molecules "click" into tetrahedral arrangements. Apparently, SAPT-5s does not discriminate accurately enough between the tetrahedral arrangements of molecules and other types of configurations. Such a conjecture is consistent with the observations made in Refs. 18 and 35, where the accuracy of SAPT-5s was shown to be uneven for different angular configurations of the dimer. The greater depth of CC-pol compared to SAPT-5s is not the main factor, as we have checked by scaling SAPT-5s which did not lead to any improvements of predictions for liquid water.

\section{E. Quantum and monomer-flexibility effects}

Although the agreement of RDFs predicted by the CC-pol+NB force field with RDFs from the experimental case (b) is very good, the contributions from the effects neglected in our simulations are almost certainly larger than the discrepancies with this experiment. First, water molecules are treated as classical objects in our simulations, which is not entirely justified at ambient temperatures, as indicated by the large magnitude of quantum contribution to the second virial coefficient, see Sec. III. Second, monomer-flexibility effects which were neglected by us may play a role in structure determination.

The quantum effects have been studied in a number of papers by performing path integral MC (PIMC), ${ }^{87-89}$ path integral MD (PIMD), ${ }^{90-92}$ or so-called centroid MD simulations. ${ }^{93-101}$ Earlier simulations used empirical potentials, ${ }^{87,93,94,102}$ which is not completely appropriate as the empirical potentials already effectively account for the quantum motions. Nevertheless, the changes of the RDFs predicted by such simulations are relatively close to those predicted by recent simulations with nonempirical potentials..$^{90,103,104}$ In particular, the very recent work of Pae- 
TABLE V. Discrepancies from the average of experiments (a) and (b) of Ref. 51 at the characteristic points on water radial distribution functions. The consecutive numbers are $\Delta r$ and $\Delta g_{\mathrm{XY}}$.

\begin{tabular}{|c|c|c|c|c|c|c|c|c|c|c|}
\hline & \multicolumn{3}{|c|}{$g_{\mathrm{OO}}$} & \multicolumn{3}{|c|}{$g_{\mathrm{OH}}$} & \multicolumn{3}{|c|}{$g_{\mathrm{HH}}$} & \multirow[b]{2}{*}{ rsmd } \\
\hline & $\max _{1}$ & $\min _{1}$ & $\max _{2}$ & $\max _{1}$ & $\min _{1}$ & $\max _{2}$ & $\max _{1}$ & $\min _{1}$ & $\max _{2}$ & \\
\hline CC-pol+NB & $0.04 /-0.08$ & $0.02 / 0.06$ & $0.17 /-0.09$ & $0.08 / 0.18$ & $0.05 / 0.09$ & $-0.02 / 0.07$ & $0.04 / 0.15$ & $0.14 / 0.04$ & $0.05 / 0.00$ & $0.084 / 0.099$ \\
\hline $\mathrm{CC}-$ pol+NB(ind) & $-0.01 /-0.01$ & $-0.13 / 0.00$ & $0.32 /-0.05$ & $0.03 / 0.28$ & $0.00 /-0.02$ & $-0.02 / 0.04$ & $0.09 / 0.17$ & $0.04 /-0.03$ & $0.00 /-0.02$ & $0.120 / 0.112$ \\
\hline TIP4P ${ }^{\mathrm{a}}$ & $-0.03 / 0.22$ & $-0.16 /-0.06$ & $0.03 / 0.00$ & $0.03 / 0.35$ & $0.03 /-0.03$ & $-0.10 / 0.00$ & $0.00 /-0.07$ & $0.03 /-0.04$ & $-0.10 /-0.01$ & $0.075 / 0.142$ \\
\hline SAPT- $5 s+N B^{b}$ & $0.03 /-0.29$ & $0.51 / 0.01$ & $0.52 /-0.12$ & $0.08 / 0.02$ & $0.05 / 0.08$ & $-0.02 / 0.04$ & $0.14 / 0.10$ & $0.19 / 0.07$ & $0.10 /-0.04$ & $0.260 / 0.117$ \\
\hline VRT(ASP-W)III ${ }^{\mathrm{c}}$ & $-0.02 /-0.46$ & $0.06 /-0.07$ & $0.10 /-0.12$ & $0.10 / 0.00$ & $-0.03 / 0.26$ & $-0.25 /-0.18$ & $0.07 / 0.04$ & $0.06 /-0.04$ & $-0.05 /-0.06$ & $0.105 / 0.194$ \\
\hline TTM2.1 $\mathrm{CM}^{\mathrm{d}}$ & $0.01 / 0.13$ & $-0.21 /-0.14$ & $-0.11 / 0.04$ & $0.03 / 0.40$ & $0.03 /-0.09$ & $-0.02 / 0.06$ & $0.03 / 0.02$ & $-0.01 /-0.11$ & $0.00 / 0.03$ & $0.081 / 0.157$ \\
\hline TTM2.1 QM ${ }^{\mathrm{d}}$ & $0.01 /-0.10$ & $-0.21 /-0.02$ & $0.08 /-0.02$ & $0.07 / 0.15$ & $0.01 /-0.03$ & $0.02 /-0.01$ & $0.09 /-0.11$ & $0.08 /-0.02$ & $0.08 / 0.00$ & $0.092 / 0.072$ \\
\hline
\end{tabular}

${ }^{\mathrm{a}}$ Reference 8 .

${ }^{\mathrm{b}}$ Reference 2 .

${ }^{c}$ Reference 53. Since it is the solid line in Fig. 3(b) of Ref. 53 which is close to the experiment (Ref. 51), we assumed that the broken line was obtained with VRT(ASP-W)III.

${ }^{\mathrm{d}}$ Reference 104 with the flexible-monomer potential of Ref. 39 .

sani et al. ${ }^{104}$ has shown that the peaks and minima in RDF curves tend to become flatter as quantum effects are turned on, while their positions remain essentially unchanged or shift slightly outward. In particular, the value of $g_{\mathrm{OO}}$ at the first maximum, first minimum, and the second maximum changed by $8.5 \%, 16 \%$, and $5.5 \%$, respectively. These changes roughly correspond to the increase of the temperature of simulation by $30^{\circ}$. Simulations with empirical potentials found similar, but somewhat stronger trends, corresponding to about $50^{\circ}$ increase. If the quantum corrections computed by Paesani et al. ${ }^{104}$ were added to our CC-pol $+\mathrm{NB} g_{\text {OO }}$ curve, this would worsen the current very close agreement with the case (b) of experiment.

Whereas the quantum effects would make the CC-pol $+\mathrm{NB}$ water less structured, the monomer-flexibility effects should not only cancel this shift but make the liquid overall more structured. The monomer-flexibility effects have been studied with empirical potentials since the 1980s. ${ }^{7,105}$ All such studies have found that these effects enhance the peaks and minima of the RDFs. In particular, the simulations with the TIP4F (Ref. 106) potential demonstrated that the major difference in the $g_{\mathrm{OO}}$ function compared to the rigidmonomer TIP4P potential ${ }^{8}$ is the deepening of the first minimum by about $10 \%$ and its shift by about $0.1 \AA$ to the left. By contrast, the first peak remains essentially unchanged (slightly shifted to the left). Monomer-flexibility effects have also been studied with $a b$ initio potentials, starting from simulations with the rigid NCC (Refs. 107 and 108) and the flexible NCC-vib (Ref. 109) potentials and found very similar relations to those observed later in Ref. 106. More recently, the monomer-flexibility effects were studied in CarParrinello simulations. ${ }^{110}$ This work found shifts in the same direction as the investigations discussed above, but somewhat more pronounced: the first minimum was lowered by about 0.2 or $35 \%$ and shifted to the left by $0.2 \AA$, whereas the first peak increased by $20 \%$ and the second one by $25 \%$.

One may now try to estimate-based on literature results-how the CC-pol+NB $g_{\mathrm{OO}}$ would change if the quantum and monomer-flexibility effects were included. The position of the first peak would remain the same and the height would increase by about $10 \%$, bringing it to a com- plete agreement with the case (b) experimental $g_{\mathrm{OO}}$. The first minimum would shift by about $0.2 \AA$ to the left and get deeper by about $20 \%$, bringing it to an excellent agreement with the case (a) $g_{\text {OOO }}$. The uncorrected position of the second peak is already in a much better agreement with case (a) than with case (b). If the size of this peak increases by about $20 \%$, it is also brought to a very good height agreement with case (a). Thus, our work may suggest that experiment (b) is more accurate than experiment (a) in the region of the first peak, but that the opposite is true for larger $r$.

\section{F. Comparison to literature radial distribution functions}

In order to compare quantitatively the RDFs from simulations with the CC-pol+NB force field to results of other simulations, one needs some reference RDFs. However, the experimental cases (a) and (b) are too different from one another to be used as such a reference. Therefore, we have decided to make comparisons relative to the average of the (a) and (b) RDFs. We have computed the deviations both in the magnitude and in the radial positions between theoretical predictions and the averaged experiment at three characteristic points on each curve: the first and second maxima and the first minimum. The results are compared in Table V. Out of many published empirical potentials for water, we have chosen TIP4P (Ref. 8) as the representative of this type of potentials since it is probably the most often used model of liquid water. The results of Table V show that the CC-pol $+\mathrm{NB}$ force field gives the rmsds for the $g_{\mathrm{XY}}$ functions as good as does TIP4P: the positions of the characteristic points are predicted by $0.009 \AA$ better by TIP4P, but the amplitudes are predicted significantly better by CC-pol $+\mathrm{NB}$ with the percentage error relative to the typical value of $g_{\mathrm{XY}}=1$ being $4.3 \%$ smaller. Thus, our purely ab initio approach recovers the RDFs equally well as empirical potentials fitted to liquid water data. The CC-pol+NB(ind) force field predicts the RDFs only slightly worse than does CC-pol+NB. It gives amplitudes which are by 3\% more accurate than those of TIP4P and positions which are $0.045 \AA$ worse. The rmsd of $\Delta g_{\mathrm{XY}}$ for the SAPT-5s+NB model of Ref. 2 is also better than that of TIP4P, despite the problems with the $g_{\mathrm{OO}}$ func- 
tion predicted by this model that were discussed above. However, these problems are visible in the rmsd of $\Delta r$ which is the largest of all potentials considered in Table V. The predictions of the VRT(ASP-W)III potential are quite good for $\Delta r$, but for $\Delta g_{\mathrm{XY}}$ are notably less accurate than those of the potentials discussed above, with the error as large as 19.4\%. The TTM2.1 flexible-monomer potential of Ref. 39 used in classical mechanics simulations ${ }^{104}$ gives the rmsd for $\Delta r 0.003 \AA$ better than does CC-pol+NB, but the rmsd of $\Delta g_{\mathrm{XY}}$ is $5.8 \%$ worse. The former deviation gets somewhat larger when quantum effects are included, ${ }^{104}$ but the latter becomes $2.7 \%$ better than given by CC-pol+NB.

It is also appropriate to compare our results to those from Car-Parrinello MD (CPMD) simulations. ${ }^{15}$ Such simulations performed in the 1990s (Refs. 111-113) obtained reasonably good agreement with experimental RDFs for liquid water, although the comparisons were made to older experiments which were quite different from the current ones. ${ }^{51,52}$ These investigations concluded that the CPMD method provides an accurate description of liquid water from first principles. On the other hand, Sprik et al. ${ }^{113}$ have shown that the experimental RDFs are reproduced well only using the BLYP functional, whereas two other functionals tried gave much less accurate results. A much more extensive study of the effects due to the choice of the DFT functional was published recently by VandeVondele et al. ${ }^{114}$ The conclusion from this work is that CPMD can reach a reasonable description of water only upon a proper choice of the functional. Such a choice would not be possible without the prior knowledge of experimental data. It has also been realized in recent years that the results of CPMD simulations for water depend critically on some technicalities in the implementation of the method. For example, the authors of Refs. 110 and 115 performed CPMD simulations for water using the same Perdew-Burke-Ernzerhof (PBE) functional, the same cutoff of the plane wave basis set, and the same pseudopotentials. Yet, the $g_{\text {OO }}$ functions from the two papers are dramatically different. The issue of the dependence of CPMD predictions on technical details of the method has been the subject of several papers in recent years showing that the agreements with experiments reached in earlier work was fortuitous. In particular, in Refs. 116 and 117, Galli and coworkers have shown that in order to obtain agreement of CPMD simulations with simulations on the corresponding Born-Oppenheimer potentials one has to use a significantly smaller fictitious electron mass than in the previous work. Sit and Marzari ${ }^{118}$ pointed out that long equilibration and averaging times, in excess of $50 \mathrm{ps,} \mathrm{are} \mathrm{needed} \mathrm{to} \mathrm{achieve} \mathrm{con-}$ verged results. Since CPMD calculations are typically started from a configuration obtained from an equilibrated MD simulation with an empirical potential, a short simulation is also likely to resemble its empirical starting point. Sit and Marzari found that with properly performed simulations using the PBE functional, the freezing point of water was predicted to be around $400 \mathrm{~K}$. Similar points were made by Fernandez-Serra and Artacho. ${ }^{119}$ If all the discussed issues are properly taken account of, the CMPD simulations with BLYP or PBE potentials result in significantly overstructured water, with RDFs much further from experiment than for the force fields included in Table V. However, very recently, Lee and Tuckerman ${ }^{120,121}$ produced yet another turn in this development by showing that CPMD can predict very reasonable RDFs after all, provided that the calculations are performed at the complete basis set limit. These authors achieved such a limit by using a discrete variable representation basis set to expand Kohn-Sham orbitals. According to Lee and Tuckerman, ${ }^{120,121}$ all previous calculations using plane waves or Gaussian basis sets were still far from convergence. Whereas one cannot exclude that a numerically converged CPMD approach will describe liquid water reasonably accurately, this would have to involve some compensation of errors as there are inherent limits to this accuracy resulting from the inability of current semilocal DFT functionals to describe the long-range dispersion interactions (see, for example, discussions in Refs. 55 and 122). The dispersion interactions are not particularly large for water, but for the minimum configuration of the water dimer do contribute about $-2 \mathrm{kcal} / \mathrm{mol}$ (Ref. 14) to the interaction energy of about $-5 \mathrm{kcal} / \mathrm{mol}$. Thus, it would be surprising if any DFT functional could predict spectra of the water dimer anywhere near the accuracy ranges discussed in Sec. II.

\section{G. Potential energy and diffusion coefficient}

In Table VI, we present the values of the average potential energy and the self-diffusion coefficient resulting from simulations based on the CC-pol potential and compare them to those resulting from the SAPT-5s potential as well as to their experimental counterparts. The numbers of molecules in the simulation box and the average simulated temperatures are also shown. The average potential energy is split into the two-body and nonadditive components. The average potential energies from both two-body simulations are in very good agreement with each other and about $1.1 \mathrm{kcal} / \mathrm{mol}$ less negative than the experimental value. Inclusion of manybody forces changes the energy of the simulated liquid by similar amounts for both potentials, increasing the binding by slightly more than $20 \%$ compared to the two-body simulation. One exception is the $\mathrm{CC}$-pol $+\mathrm{NB}$ (ind) result, which is $27 \%$ more negative than the CC-pol energy. Most of the additional binding is brought by the three-body nonadditive interactions. The energetic effect of the $N>$ three-body terms in the potential is much smaller, even though, as discussed above, these terms significantly change the structure of the liquid in the CC-pol case. It should be noted that the net increase in total binding energy is in fact a sum of two contributions: the actual contribution of many-body interactions, shown in column 5 of Table VI, and the change in the average two-body interaction (column 4) with respect to the purely two-body simulation (shown in the first row of Table VI). The latter change, arising from the geometry rearrangement brought about by introduction of many-body forces, ranges between -0.1 and $-0.3 \mathrm{kcal} / \mathrm{mol}$. Only the SAPT-5s $+\mathrm{NB}$ (ind) simulation leads to a positive change $(0.07 \mathrm{kcal} / \mathrm{mol})$ in the two-body contribution. Interestingly, the size of the rearrangement effect is, in most cases, approximately the same for CC-pol and SAPT-5s potentials, although the liquid structures are quite different. 
TABLE VI. Average potential energy (in $\mathrm{kcal} / \mathrm{mol}$ ), coordination number, and self-diffusion coefficient (in $10^{-5} \mathrm{~cm}^{2} / \mathrm{s}$ ) of ambient water obtained from MD simulations with various potentials.

\begin{tabular}{|c|c|c|c|c|c|c|c|}
\hline \multirow[b]{2}{*}{ Potential } & \multirow[b]{2}{*}{$N$} & \multirow[b]{2}{*}{$T / \mathrm{K}$} & \multicolumn{3}{|c|}{ Energy } & \multirow[b]{2}{*}{ Coord. No. } & \multirow[b]{2}{*}{$D$} \\
\hline & & & Two-body & Nonadditive & Total & & \\
\hline CC-pol (2-body) & 512 & 305 & -8.79 & 0.0 & -8.79 & 11.9 & 7.8 \\
\hline CC-pol+3B & 256 & 313 & -9.00 & -1.73 & -10.73 & 10.6 & 4.4 \\
\hline CC-pol+NB & 256 & 302 & -9.03 & -1.86 & -10.89 & 5.6 & 2.4 \\
\hline CC-pol+NB(ind) & 512 & 306 & -8.89 & -2.31 & -11.20 & 4.7 & 2.0 \\
\hline SAPT-5s (2-body) ${ }^{\mathrm{a}}$ & 128 & 285 & -8.84 & 0.0 & -8.84 & 11.3 & 5.6 \\
\hline SAPT $-5 s+3 B^{a}$ & 128 & 301 & -9.10 & -1.54 & -10.64 & 7.9 & 4.2 \\
\hline $\mathrm{SAPT}-5 \mathrm{~s}+\mathrm{NB}^{\mathrm{a}}$ & 128 & 308 & -9.07 & -1.69 & -10.76 & 8.6 & 4.2 \\
\hline SAPT $-5 s+N B($ ind $)$ & 512 & 300 & -8.77 & -1.96 & -10.73 & & 3.5 \\
\hline Experiment & & 298.15 & & & $-9.92 \pm 0.3^{\mathrm{b}}$ & $4.5,6.0^{c}$ & $2.3^{\mathrm{d}}$ \\
\hline
\end{tabular}

${ }^{a}$ Energies from NVT Monte Carlo simulations of Ref. 2 with 512 molecules at $T=298.15 \mathrm{~K}$; self-diffusion coefficient obtained in this work.

${ }^{\mathrm{b}}$ Reference 123, value obtained from experimental vaporization enthalpy in $298.15 \mathrm{~K}$, equal to $10.51 \mathrm{kcal} / \mathrm{mol}$ by subtracting $R T=0.59 \mathrm{kcal} / \mathrm{mol}$ (and multiplying the result by -1 ). See Ref. 124 for a discussion of the error estimate.

${ }^{\mathrm{c}}$ Computed from the RDFs of Soper, Ref. 51.

${ }^{\mathrm{d}}$ Mills, Ref. 125.

When comparing the simulated average potential energies to the experimental estimate, one should bear in mind that our simulations neglect the quantum effects, which are quite important at ambient temperature. The quantum simulation data ${ }^{87,90,93,94}$ place the quantum correction to the potential energy of the liquid in the range from +0.7 to $+1.5 \mathrm{kcal} / \mathrm{mol}$. The recent simulations by Paesani et al. ${ }^{104}$ gave a difference of $1.05 \mathrm{kcal} / \mathrm{mol}$. Upon addition of the quantum correction to the results of our simulations with nonadditive forces included, the agreement with experiment is greatly improved. For example, adding to the classical $\mathrm{CC}-\mathrm{pol}+\mathrm{NB}$ value of $-10.89 \mathrm{kcal} / \mathrm{mol}$ the $1.05 \mathrm{kcal} / \mathrm{mol}$ correction from Ref. 104, we get $-9.84 \mathrm{kcal} / \mathrm{mol}$, only $0.08 \mathrm{kcal} / \mathrm{mol}$ from experiment and well within the estimated error bars of the experimental value. ${ }^{123,124}$ For comparison, TIP4P gives ${ }^{93}-9.81$ and $-8.95 \mathrm{kcal} / \mathrm{mol}$ at the classical and quantum levels, respectively. Our predictions with or without the quantum correction are closer to experiment than the predictions of the TTM2.1-F potential. ${ }^{104}$

The self-diffusion coefficient $D$ was estimated using the Einstein relation

$$
D=\lim _{t \rightarrow \infty} \frac{1}{6 t}\left\langle|r(t)-r(0)|^{2}\right\rangle,
$$

i.e., as the slope of the average square displacement of the center of mass of a molecule as a function of time, calculated using data corresponding to times longer than $1 \mathrm{ps}$. The values of $D$ reflect the mobility of molecules and correlate well with the structural features inferred from Figs. 7 and 8. Both the CC-pol and SAPT-5s pure two-body simulations fail to generate a hydrogen-bonded network and result in $D$ values 2-3 times larger than the experimental value. When the three-body interactions are turned on, the structure becomes more rigid, which leads to lower mobility and thus lower $D$. Inclusion of $N>$ three-body induction interactions brings the $\mathrm{CC}-$ pol + NB [as well as CC-pol+ $\mathrm{NB}$ (ind)] self-diffusion coefficient to a very good agreement with the measured value of $2.30 \times 10^{-5} \mathrm{~cm}^{2} / \mathrm{s}$ at $T=298.2 \mathrm{~K}$ (Ref. 125) or 2.55 $\times 10^{-5} \mathrm{~cm}^{2} / \mathrm{s}$ after an interpolation to $T=302 \mathrm{~K}$ reached by our simulations. On the other hand, the SAPT $-5 \mathrm{~s}+\mathrm{NB}$ value is practically the same as the SAPT- $5 s+3 \mathrm{~B}$ one, consistent with the structural similarity observed for both sets of simulations. It should be noted that the computed values of the diffusion coefficient would most likely increase if quantum effects were included. Based on quantum simulations with both empirical potentials ${ }^{93,94}$ and nonempirical potentials, ${ }^{104}$ this increase at ambient temperature amounts to a factor of about 1.5. Thus, quantum simulations with CC-pol+NB may overestimate $D$ by about $50 \%$. For comparison, the classical and quantum values of $D$ from the TIP4P potential are 3.6 and $5.5 \times 10^{-5} \mathrm{~cm}^{2} / \mathrm{s}$, respectively. ${ }^{93}$ As mentioned before, one should rather compare to classical calculations with empirical potentials and thus the estimated CC-pol+NB quantum $D$ happens to coincide with the TIP4P classical value. The calculations with the TTM2.1-F potential gave ${ }^{104}$ the classical and quantum values of $D$ equal to 1.50 and 2.25 $\times 10^{-5} \mathrm{~cm}^{2} / \mathrm{s}$, closer to experiment than the estimated quantum value from CC-pol+NB (only 2.2\% error). However, the difference is really smaller than this comparison may suggest. First, since TTM2.1-F includes many-body effects only via a polarization term, one should compare these results to the value obtained in the CC-pol+NB(ind) simulation, which after scaling for quantum effects becomes equal to 3.0 $\times 10^{-5} \mathrm{~cm}^{2} / \mathrm{s}$. Second, the diffusion coefficient is a sensitive function of temperature and our CC-pol+NB(ind) simulations ended up in the temperature of $306 \mathrm{~K}$. The interpolated experimental value ${ }^{125}$ at this temperature is 2.8 $\times 10^{-5} \mathrm{~cm}^{2} / \mathrm{s}$. Thus, the CC-pol+NB(ind) prediction after the quantum correction is only in $7 \%$ error. Of course, also the monomer-flexibility effects will change the diffusion coefficient. We could not find any reliable estimates of this effect (see, however, Ref. 110), but since water with rigid monomers is less structured, as discussed above, the more structured water with flexible monomers will have a lower diffusion coefficient. Thus, this effect may well cancel the increase due to the quantum effect. 


\section{CONCLUSIONS}

The CC-pol potential of Ref. 13 was used in converged six-dimensional variational calculations of the bound intermolecular rovibrational levels of both $\left(\mathrm{H}_{2} \mathrm{O}\right)_{2}$ and $\left(\mathrm{D}_{2} \mathrm{O}\right)_{2}$. The transition frequencies-including very low-energy transitions between the levels split by tunneling-provide a critical test of water pair potentials when compared to the data from high-resolution spectroscopy. It turns out that the $\mathrm{CC}$ pol potential performs substantially better than any older $a b$ initio potential, also better than the recently published SDFT-5s potential. ${ }^{35}$ Even potentials that were explicitly fitted to the spectral data, such as the different versions of the VRT(ASP) potential or the VRT(MCY-5f) potential, ${ }^{11,42,43}$ or were improved with the use of these data such as SAPT-5st, ${ }^{28}$ do not give uniformly better agreement with the measured data than $\mathrm{CC}$-pol. The remaining small discrepancies between the theoretical and experimental values of the ground state tunneling splittings are probably mainly due to the fact that the CC-pol potential does not account for the effects of monomer flexibility. Interestingly, the intermolecular mode $\mathrm{D}_{2} \mathrm{O}$ dimer frequencies from the $\mathrm{CC}$-pol potential are better than those from the VRT(ASP-W)III potential ${ }^{42}$ which was fitted to these frequencies. For the $\mathrm{H}_{2} \mathrm{O}$ dimer, CC-pol spectra are overall significantly closer to experiment than those from VRT(ASP-W)III.

The second virial coefficient computed from the CC-pol surface over a wide range of temperatures is in very good agreement with the experimental correlation of Harvey and Lemmon. ${ }^{29}$ For temperatures below $500 \mathrm{~K}$, the accuracy of the CC-pol results surpasses that of the SAPT-5s ones, ${ }^{30}$ while the latter are closer to experiment in the hightemperature regime. However, in view of the scarcity of experimental data for higher temperature, the values computed from SAPT-5s have actually been used as data points in the experimental correlation. ${ }^{29}$ Since the CC-pol surface is more accurate than SAPT-5s, we believe that the virial data computed using the former surface are more accurate for all temperatures. Any remaining discrepancies from the exact values are most likely caused by the neglect of monomer flexibility and-for the lowest temperatures-by residual inaccuracies in the treatment of quantum effects.

The CC-pol potential also allowed to shed light onto the problem of the convergence of the many-body expansion of the interaction energy in liquid water. The MD simulations of the liquid were performed at ambient conditions, with $\mathrm{CC}$ pol as a pair potential, the three-body nonadditive effects represented by the potential of Ref. 48 , and the $N>3$ nonadditive induction effects given by the classical asymptotic polarization model (implemented via the explicit polarization term in CC-pol). The simulations revealed that the manybody effects are responsible for $17 \%-20 \%$ of the total potential energy in the liquid, and most of this effect is a consequence of three-body interactions. These results are in agreement with previous studies ${ }^{2}$ based on the SAPT-5s pair potential. Quite different conclusions are drawn concerning the liquid structure. In simulations with the SAPT-5s potential, the three-body interactions were found to account well for the positions and magnitudes of the maxima and minima in the $g_{\mathrm{OH}}$ and $g_{\mathrm{HH}}$ radial distribution functions. However, the first minimum and second peak in the $g_{\mathrm{OO}}$ function were reproduced poorly, indicating lack of tetrahedral structure in the simulated liquid. Virtually no improvement was observed upon the inclusion of $N>$ three-body induction effects; i.e., the structure was saturated already at the three-body level. In the case of the present CC-pol simulations, both the threebody and higher nonadditive effects significantly influence the liquid structure. When all these effects are accounted for, the shape of the $g_{\mathrm{OO}}$ function is brought to very good agreement with experiment, indicating the onset of a tetrahedral network in the liquid. Thus, the tetrahedral structure of liquid water effectively results from pairwise nonadditive interactions. Our calculations show that relatively small changes in the potential make the molecules click into tetrahedral, hydrogen bonded arrangements from more disoriented structures.

Our results allow us to make very general conclusions about the convergence of the many-body expansion for liquid water. It can be considered to be a difficult system since the expansion cannot be truncated at the three-body level, as it can be done for liquid argon. ${ }^{50}$ Fortunately, it appears that the nonadditive four- and higher-body contributions are well represented by the asymptotic induction effects. Therefore, with sufficiently accurate two- and three-body potentials, one can achieve reliable predictions for bulk water after approximating higher-body effects by the induction model. The latter effects increase the magnitude of the total average interaction energy by only $0.16 \mathrm{kcal} / \mathrm{mol}$ or $1.5 \%$, nevertheless have a qualitative impact on the $g_{\mathrm{OO}} \mathrm{RDF}$. The relative energetic contributions of individual many-body terms obtained by us are similar to those derived from studies of small clusters, see the discussion of this issue in Ref. 1.

Although the $g_{\mathrm{XY}}$ functions from the CC-pol+NB simulations do not include quantum and monomer flexibility effects, the agreement with experiments ${ }^{51,52}$ is very good. This is partly due to the fact that the two types of effects cancel to some extent. If the quantum and monomer-flexibility corrections estimated from literature calculations are added to the computed CC-pol+NB $g_{\text {OO }}$ function, this results in an excellent agreement with experiment (b) (Ref. 51) in the region of the first peak and with experiment (a) (Ref. 51) for larger $r$. Thus, our results may suggest that the two experiments have different accuracies in these two regions. Also, with or without corrections, theory quite consistently predicts a larger height of the first peak in the $g_{\mathrm{OH}}$ function, indicating a potential experimental inaccuracy in this region. If the estimates of monomer-flexibility effects from Ref. 110 are included, the disagreement becomes rather dramatic.

The CC-pol+NB predictions of $g_{\mathrm{XY}}$ 's are overall notably better than those given by the SAPT-5st and VRT(ASP-W)III potentials. This observation together with the comparisons made earlier about dimer spectra and virial coefficients show that the CC-pol potential predicts the overall properties of water better than the best semiempirical potentials fitted to spectral data for the water dimer. ${ }^{28,42,43}$ Since empirical potentials of this type can be obtained only for specific systems with a large number of transitions measured and assigned-in fact, the water dimer is one of the very few 
systems for which such potentials exist-an ab initio approach offers now a more expeditious way of arriving at such potentials, even for systems much larger than water, such as the benzene dimer ${ }^{32}$ or the cyclotrimethylene trinitramine dimer. $^{33}$

The values of the self-diffusion coefficient have been calculated for different interaction models. These values are consistent with the structural features, reflecting a smaller mobility of molecules involved in tetrahedral networks compared to those in an unstructured liquid. The (classical) value given by the CC-pol+NB force field agrees well with experiment, although neglected quantum effects would probably increase this value by about $50 \%$.

The overall agreement of liquid water properties predicted by the CC-pol+NB force field with experiment is comparable to that achieved by the empirical potentials such as TIP4P (Ref. 8) which were fitted to these properties. The overall discrepancies of TIP4P $g_{\mathrm{XY}}$ 's from experiment are actually slightly larger than those produced by $\mathrm{CC}-$ pol+NB. After estimated quantum corrections are included, CC-pol $+\mathrm{NB}$ predicts the internal energy and the diffusion coefficient nearly identical to those predicted by TIP4P in classical calculations. We believe this is an excellent performance for a force field which was obtained purely $a b$ initio, without use of any experimental data. Moreover, none of the existing empirical force fields of this type can simultaneously reproduce water dimer spectra or virial coefficients.

Our CC-pol force field works well for a broad range of properties and in fact in all cases (dimer vibrational transitions, rotational constants, tunneling splittings, virial coefficients, and properties of liquid water) provides an accuracy comparable to that achievable with empirical potentials fitted to specific properties (either spectral or bulk). Our potential also predicts all the dimer properties better than any published $a b$ initio potential and on par with the best empirical potentials fitted to the spectra. In across-the-board comparison to the TTM2.1-F potential, CC-pol predicts the spectral properties of the dimer with about three to eight times smaller errors. The virial coefficients of TTM2.1-F are unknown, but its predecessor, TTM2-R potential, gives for higher temperatures four times larger errors than does $\mathrm{CC}$ pol. The RDFs from TTM2.1-F classical simulations are less accurate than those given by $\mathrm{CC}$-pol+NB if compared to the average of the experiments (a) and (b). The quantum simulations with TTM2.1-F give a slightly larger rmsd error than $\mathrm{CC}$-pol $+\mathrm{NB}$ in positions of the peaks, but agree slightly better in predictions of the amplitudes.

Since the improvements of the present results over those obtained with the SAPT-5s potential are due only to the better quality of the pair potential, one may think that the reason is the use of the CCSD(T) method instead of SAPT to compute $a b$ initio points. This turned out not to be the case; the major reason for improvements is a much better basis set employed in the development of CC-pol (including complete basis set extrapolations); see the discussion in Ref. 18.

It is worth pointing out that the consecutive steps in the development of the water potentials from our group lead to a systematic improvement of the agreement with experiment. The first potential SAPT-pp (Ref. 14) provided excellent agreement with the experimental second virial coefficients but the (unpublished) dimer spectra were less accurate than those from SAPT-5s. SAPT-pp was also too time consuming to be used in molecular simulations. The SAPT-5s potential, which differed from SAPT-pp mainly by increasing the number of grid points and a different form of the fitting function, ${ }^{26,30}$ gave very accurate spectra, ${ }^{26,28}$ but when used together with the SAPT three-body potential, ${ }^{48}$ it gave unsatisfactory predictions for the structure of water. ${ }^{2}$ The present improvement of the pair potential, mainly due to the use of large basis sets, solved the latter problem and at the same time resulted in improved spectra.

\section{ACKNOWLEDGMENTS}

This work was supported by the NSF Grant Nos. CHE0239611 and CHE-0555979.

${ }^{1}$ K. Szalewicz, R. Bukowski, and B. Jeziorski, in Theory and Applications of Computational Chemistry: The First 40 Years. A Volume of Technical and Historical Perspectives, edited by C. E. Dykstra, G. Frenking, K. S. Kim, and G. E. Scuseria (Elsevier, Amsterdam, 2005), Chap. 33, pp. 919-962.

${ }^{2}$ E. M. Mas, R. Bukowski, and K. Szalewicz, J. Chem. Phys. 118, 4404 (2003).

${ }^{3}$ V. F. Lotrich and K. Szalewicz, J. Phys. Chem. 106, 9688 (1997).

${ }^{4}$ V. F. Lotrich and K. Szalewicz, Phys. Rev. Lett. 79, 1301 (1997).

${ }^{5}$ V. F. Lotrich and K. Szalewicz, J. Chem. Phys. 112, 112 (2000).

${ }^{6}$ V. F. Lotrich, P. Jankowski, and K. Szalewicz, J. Chem. Phys. 108, 4725 (1998)

${ }^{7}$ J. R. Reimers, R. O. Watts, and M. L. Klein, Chem. Phys. 64, 95 (1982).

${ }^{8}$ W. L. Jorgensen, J. Chandrasekhar, J. D. Madura, R. W. Impey, and M. L. Klein, J. Chem. Phys. 79, 926 (1983).

${ }^{9}$ H. J. C. Berendsen, J. R. Grigera, and T. P. Straatsma, J. Phys. Chem. 91, 6209 (1987).

${ }^{10}$ M. W. Mahoney and W. L. Jorgensen, J. Chem. Phys. 112, 8910 (2000).

${ }^{11}$ R. S. Fellers, L. B. Braly, R. J. Saykally, and C. Leforestier, J. Chem. Phys. 110, 6306 (1999).

${ }^{12}$ H. Chen, S. Liu, and J. C. Light, J. Chem. Phys. 110, 168 (1999).

${ }^{13}$ R. Bukowski, K. Szalewicz, G. C. Groenenboom, and A. van der Avoird, Science 315, 1249 (2007).

${ }^{14}$ E. M. Mas, K. Szalewicz, R. Bukowski, and B. Jeziorski, J. Chem. Phys. 107, 4207 (1997)

${ }^{15}$ R. Car and M. Parrinello, Phys. Rev. Lett. 55, 2471 (1985).

${ }^{16}$ R. N. Barnett and U. Landman, Phys. Rev. B 48, 2081 (1993).

${ }^{17}$ D. Xenides, B. R. Randolf, and B. M. Rode, J. Mol. Liq. 123, 2 (2006).

${ }^{18}$ R. Bukowski, K. Szalewicz, G. C. Groenenboom, and A. van der Avoird, J. Chem. Phys. 128, 094313 (2008).

${ }^{19}$ E. Zwart, J. J. ter Meulen, and W. L. Meerts, J. Mol. Spectrosc. 173, 115 (1990).

${ }^{20}$ E. Zwart, J. J. ter Meulen, W. L. Meerts, and L. H. Coudert, J. Mol. Spectrosc. 147, 27 (1991).

${ }^{21}$ G. T. Fraser, Int. Rev. Phys. Chem. 10, 189 (1991).

${ }^{22}$ E. N. Karyakin, G. T. Fraser, and R. D. Suenram, Mol. Phys. 78, 1179 (1993).

${ }^{23}$ J. B. Paul, R. A. Provencal, and R. J. Saykally, J. Phys. Chem. A 102, 3279 (1998)

${ }^{24}$ L. B. Braly, J. D. Cruzan, K. Liu, R. S. Fellers, and R. J. Saykally, J. Chem. Phys. 112, 10293 (2000).

${ }^{25}$ L. B. Braly, K. Liu, M. G. Brown, F. N. Keutsch, R. S. Fellers, and R. J. Saykally, J. Chem. Phys. 112, 10314 (2000).

${ }^{26}$ G. C. Groenenboom, E. M. Mas, R. Bukowski, K. Szalewicz, P. E. S. Wormer, and A. van der Avoird, Phys. Rev. Lett. 84, 4072 (2000).

${ }^{27}$ M. J. Smit, G. C. Groenenboom, P. E. S. Wormer, A. van der Avoird, R. Bukowski, and K. Szalewicz, J. Phys. Chem. A 105, 6212 (2001).

${ }^{28}$ G. C. Groenenboom, P. E. S. Wormer, A. van der Avoird, E. M. Mas, R. Bukowski, and K. Szalewicz, J. Chem. Phys. 113, 6702 (2000).

${ }^{29}$ A. H. Harvey and E. W. Lemmon, J. Phys. Chem. Ref. Data 33, 369 (2004).

${ }^{30}$ E. M. Mas, R. Bukowski, K. Szalewicz, G. C. Groenenboom, P. E. S. 
Wormer, and A. van der Avoird, J. Chem. Phys. 113, 6687 (2000).

${ }^{31}$ R. Bukowski, K. Szalewicz, and C. Chabalowski, J. Phys. Chem. A 103 , 7322 (1999)

${ }^{32}$ R. Podeszwa, R. Bukowski, and K. Szalewicz, J. Phys. Chem. A 110, 10345 (2006).

${ }^{33}$ R. Podeszwa, R. Bukowski, B. M. Rice, and K. Szalewicz, Phys. Chem. Chem. Phys. 9, 5561 (2007).

${ }^{34}$ C. Millot and A. J. Stone, Mol. Phys. 77, 439 (1992).

${ }^{35}$ R. Bukowski, K. Szalewicz, G. C. Groenenboom, and A. van der Avoird, J. Chem. Phys. 125, 044301 (2006).

${ }^{36}$ C. Millot, J. C. Soetens, M. T. C. M. Costa, M. P. Hodges, and A. J. Stone, J. Phys. Chem. 102, 754 (1998)

${ }^{37}$ C. J. Burnham and S. S. Xantheas, J. Chem. Phys. 116, 1500 (2002).

${ }^{38}$ C. J. Burnham and S. S. Xantheas, J. Chem. Phys. 116, 5115 (2002).

${ }^{39}$ G. S. Fanourgakis and S. S. Xantheas, J. Phys. Chem. A 110, 4100 (2006).

${ }^{40}$ M. Torheyden and G. Jansen, Mol. Phys. 104, 2101 (2006).

${ }^{41}$ R. S. Fellers, C. Leforestier, L. B. Braly, M. G. Brown, and R. J. Saykally, Science 284, 945 (1999).

${ }^{42}$ N. Goldman, R. S. Fellers, M. G. Brown, L. B. Braly, C. J. Keoshian, C. Leforestier, and R. J. Saykally, J. Chem. Phys. 116, 10148 (2002).

${ }^{43}$ C. Leforestier, F. Gatti, R. Fellers, and R. Saykally, J. Chem. Phys. 117, 8710 (2002)

${ }^{44}$ F. N. Keutsch, N. Goldman, H. A. Harker, C. Leforestier, and R. J. Saykally, Mol. Phys. 101, 3477 (2003).

${ }^{45}$ F. N. Keutsch, L. B. Braly, M. G. Brown, H. A. Harker, P. B. Petersen, C. Leforestier, and R. J. Saykally, J. Chem. Phys. 119, 8927 (2003).

${ }^{46}$ O. Matsuoka, E. Clementi, and M. Yoshimine, J. Chem. Phys. 64, 1351 (1976).

${ }^{47}$ C. Leforestier, L. B. Braly, K. Liu, M. J. Elrod, and R. J. Saykally, J. Chem. Phys. 106, 8527 (1997).

${ }^{48}$ E. M. Mas, R. Bukowski, and K. Szalewicz, J. Chem. Phys. 118, 4386 (2003)

${ }^{49}$ V. F. Lotrich and K. Szalewicz, J. Chem. Phys. 106, 9668 (1997).

${ }^{50}$ R. Bukowski and K. Szalewicz, J. Chem. Phys. 114, 9518 (2001).

${ }^{51}$ A. K. Soper, Chem. Phys. 258, 121 (2000).

${ }^{52}$ G. Hura, J. M. Sorenson, R. M. Glaeser, and T. Head-Gordon, J. Chem. Phys. 113, 9140 (2000).

${ }^{53}$ N. Goldman, C. Leforestier, and R. J. Saykally, Philos. Trans. R. Soc. London, Ser. A 363, 493 (2005).

${ }^{54}$ A. J. Misquitta, R. Podeszwa, B. Jeziorski, and K. Szalewicz, J. Chem. Phys. 123, 214103 (2005).

${ }_{55}^{5}$ A. J. Misquitta and K. Szalewicz, J. Chem. Phys. 122, 214109 (2005).

${ }^{56}$ A. J. Misquitta and K. Szalewicz, Chem. Phys. Lett. 357, 301 (2002).

${ }^{57}$ S. F. Boys and F. Bernardi, Mol. Phys. 19, 553 (1970).

${ }^{58}$ F. B. van Duijneveldt, J. G. C. M. van Duijneveldt-van de Rijdt, and J. H. van Lenthe, Chem. Rev. (Washington, D.C.) 94, 1873 (1994).

${ }^{59}$ T. Korona, H. L. Williams, R. Bukowski, B. Jeziorski, and K. Szalewicz, J. Chem. Phys. 106, 5109 (1997).

${ }^{60}$ K. Szalewicz and B. Jeziorski, J. Chem. Phys. 104, 1198 (1998).

${ }^{61}$ G. Chalasinski and M. M. Szczesniak, Chem. Rev. (Washington, D.C.) 100, 4227 (2000)

${ }^{62}$ E. M. Mas and K. Szalewicz, J. Chem. Phys. 104, 7606 (1996).

${ }^{63}$ M. Jeziorska, P. Jankowski, K. Szalewicz, and B. Jeziorski, J. Chem. Phys. 113, 2957 (2000)

${ }^{64}$ G. S. Tschumper, M. L. Leininger, B. C. Hoffman, E. F. Valeev, H. F. Schaefer III, and M. Quack, J. Chem. Phys. 116, 690 (2002).

${ }^{65}$ H. C. Longuet-Higgins, Mol. Phys. 6, 445 (1963).

${ }^{66}$ L. B. Braly, Ph.D. thesis, University of California at Berkeley, 1999.

${ }^{67}$ S. Kuwajima and A. Warshel, J. Phys. Chem. 94, 460 (1990).

${ }^{68}$ Y. Scribano, N. Goldman, R. J. Saykally, and C. Leforestier, J. Phys. Chem. A 110, 5411 (2006).

${ }^{69}$ K. Szalewicz, G. Murdachaew, R. Bukowski, O. Akin-Ojo, and C. Leforestier, in Lecture Series on Computer and Computational Science: ICCMSE 2006, edited by G. Maroulis and T. Simos (Brill Academic, Leiden, 2006), Vol. 6, pp. 482-491.

${ }^{70}$ C. G. Gray and K. E. Gubbins, Theory of Molecular Fluids Volume 1: Fundamentals (Clarendon, Oxford, 1984).

${ }^{71}$ P. E. S. Wormer, J. Chem. Phys. 122, 184301 (2005).

${ }^{72}$ G. K. Schenter, J. Chem. Phys. 117, 6573 (2002).

${ }^{73}$ M. Takahashi and M. Imada, J. Phys. Soc. Jpn. 53, 3765 (1984).

${ }^{74}$ R. A. Bustos Marún, E. A. Coronado, and J. C. Ferrero, Chem. Phys. Lett. 405, 203 (2005).

${ }^{75}$ G. S. Kell, G. E. McLaurin, and E. Whalley, Proc. R. Soc. London, Ser
A 425, 49 (1989).

${ }^{76}$ CRC Handbook of Chemistry and Physics, edited by D. R. Lide (CRC, Boca Raton, FL, 1995)

${ }^{77}$ Z. Slanina, J. Radioanal. Nucl. Chem. 142, 525 (1990).

${ }^{78}$ C. J. Burnham, J. Li, S. S. Xantheas, and M. Leslie, J. Chem. Phys. 110, 4566 (1999).

${ }^{79} \mathrm{R}$. Bukowski et al. (unpublished).

${ }^{80}$ K. T. Tang and J. P. Toennies, J. Chem. Phys. 80, 3726 (1984).

${ }^{81}$ A. K. Soper, F. Bruni, and M. A. Ricci, J. Chem. Phys. 106, 247 (1997).

${ }^{82}$ P. Wernet, D. Nordlund, U. Bergmann, M. Cavalleri, M. Odelius, H. Ogasawara, L. A. Naslund, T. K. Hirsch, L. Ojanae, P. Glatzel, L. G. M. Pettersson, and A. Nilsson, Science 304, 995 (2004).

${ }^{83}$ A. Nilsson, P. Wernet, D. Nordlund, U. Bergmann, M. Cavalleri, M. Odelius, H. Ogasawara, L. A. Naslund, T. K. Hirsch, P. Glatzel, L. G. M. Pettersson, Science 308, 793a (2005).

${ }^{84}$ J. D. Smith, C. D. Cappa, K. R. Wilson, B. M. Messer, R. C. Cohen, and R. J. Saykally, Science 306, 851 (2004).

${ }^{85}$ J. D. Smith, C. D. Cappa, B. M. Messer, R. C. Cohen, and R. J. Saykally, Science 308, 793b (2005).

${ }^{86}$ R. L. Wang, H. J. Kreuzer, and M. Grunze, Phys. Chem. Chem. Phys. 8, 4744 (2006).

${ }^{87}$ G. S. D. Buono, P. J. Rossky, and J. Schnitker, J. Chem. Phys. 95, 3728 (1991).

${ }^{88}$ R. A. Kuharski and P. J. Rossky, Chem. Phys. Lett. 103, 357 (1984).

${ }^{89}$ R. A. Kuharski and P. J. Rossky, J. Chem. Phys. 103, 5164 (1985).

${ }^{90}$ H. A. Stern and B. J. Berne, J. Chem. Phys. 115, 7622 (2001).

${ }^{91}$ B. J. Berne and D. Thirumalai, Annu. Rev. Phys. Chem. 37, 401 (1986).

${ }^{92}$ M. E. Tuckerman, B. J. Berne, G. J. Martyna, and M. L. Klein, J. Chem. Phys. 97, 2635 (1992).

${ }^{93}$ L. H. de la Pena and P. G. Kusalik, J. Chem. Phys. 121, 5992 (2004).

${ }^{94}$ L. H. de la Pena and P. G. Kusalik, J. Am. Chem. Soc. 127, 5246 (2005).

${ }^{95}$ J. S. Cao and G. A. Voth, J. Chem. Phys. 100, 5093 (1994)

${ }^{96}$ J. S. Cao and G. A. Voth, J. Chem. Phys. 100, 5106 (1994).

${ }^{97}$ J. S. Cao and G. A. Voth, J. Chem. Phys. 101, 6157 (1994).

${ }^{98}$ J. S. Cao and G. A. Voth, J. Chem. Phys. 101, 6168 (1994).

${ }^{99}$ G. A. Voth, Adv. Chem. Phys. 93, 135 (1996).

${ }^{100}$ J. Lobaugh and G. A. Voth, J. Chem. Phys. 106, 2400 (1997).

${ }^{101}$ L. H. de la Pena and P. G. Kusalik, J. Phys. Chem. A 109, 7236 (2005).

${ }^{102}$ D. Marx and M. H. Müser, J. Phys.: Condens. Matter 11, R117 (1999).

${ }^{103}$ G. S. Fanourgakis, G. K. Schenter, and S. S. Xantheas, J. Chem. Phys. 125, 141102 (2006).

${ }^{104}$ F. Paesani, S. Iuchi, and G. A. Voth, J. Chem. Phys. 127, 074506 (2007).

${ }^{105}$ J. R. Reimers and R. O. Watts, Chem. Phys. 91, 201 (1984).

${ }^{106}$ M. W. Mahoney and W. L. Jorgensen, J. Chem. Phys. 115, 10758 (2001).

${ }^{107}$ U. Niessar, G. Corongiu, M.-J. Huang, M. Dupuis, and E. Clementi, Int. J. Quantum Chem., Symp. 23, 421 (1989).

${ }^{108}$ U. Niessar, G. Corongiu, E. Clementi, G. R. Kneller, and D. K. Bhattacharya, J. Phys. Chem. 94, 7949 (1990).

${ }^{109}$ G. Corongiu and E. Clementi, J. Chem. Phys. 97, 2030 (1992); 97, 8818(E) (1992)

${ }^{110}$ M. Allesch, E. Schwegler, F. Gygi, and G. Galli, J. Chem. Phys. 120, 5192 (2004)

${ }^{111}$ K. Laasonen, M. Sprik, M. Parrinello, and R. Car, J. Chem. Phys. 99, 9080 (1993)

${ }^{112}$ P. L. Silvestrelli and M. Parrinello, J. Chem. Phys. 111, 3572 (1999).

${ }^{113}$ M. Sprik, J. Hutter, and M. Parrinello, J. Chem. Phys. 105, 1142 (1996).

${ }^{114}$ J. VandeVondele, F. Mohamed, M. Krack, J. Hutter, M. Sprik, and M. Parrinello, J. Chem. Phys. 122, 014515 (2005).

${ }^{115}$ M. Sharma, Y. Wu, and R. Car, Int. J. Quantum Chem. 95, 821 (2003).

${ }^{116}$ J. C. Grossman, E. Schwegler, E. W. Draeger, F. Gygi, and G. Galli, J. Chem. Phys. 120, 300 (2004).

${ }^{117}$ E. Schwegler, J. C. Grossman, F. Gygi, and G. Galli, J. Chem. Phys. 121, 5400 (2004)

${ }^{118}$ P. H.-L. Sit and N. Marzari, J. Chem. Phys. 122, 204510 (2005).

${ }^{119}$ M. V. Fernandez-Serra and E. Artacho, J. Chem. Phys. 121, 11136 (2004).

${ }^{120}$ H.-S. Lee and M. E. Tuckerman, J. Chem. Phys. 125, 154507 (2006).

${ }^{121}$ H.-S. Lee and M. E. Tuckerman, J. Chem. Phys. 126, 164501 (2007).

${ }^{122}$ X. Wu, M. C. Vargas, S. Nayak, V. Lotrich, and G. Scoles, J. Chem. Phys. 115, 8748 (2001)

${ }^{123}$ Properties of Ordinary Water-Substance, edited by N. E. Dorsey, (Hafner, New York, 1968).

${ }^{124}$ W. L. Jorgensen, J. Chem. Phys. 77, 4156 (1982).

${ }^{125}$ R. Mills, J. Phys. Chem. 77, 685 (1973). 